\title{
Fourier reconstruction of marine-streamer data in four spatial coordinates
}

\author{
P. Zwartjes ${ }^{1}$ and A. Gisolf ${ }^{2}$
}

\begin{abstract}
Many methods exist for interpolation of seismic data in one and two spatial dimensions, but few can interpolate properly in three or four spatial dimensions. Marine multi-streamer data typically are sampled relatively well in the midpoint and absolute offset coordinates but not in the azimuth because the crossline shot coordinate is significantly under sampled. We approach the problem of interpolation of marine-streamer data in four spatial dimensions by splitting the problem into a 1D interpolation along the densely sampled streamers and a 3D Fourier reconstruction for the remaining spatial coordinates. In Fourier reconstruction, the Fourier coefficients that synthesize the nonuniformly sampled seismic data are estimated in a least-squares inversion. The method is computationally efficient, requires no subsurface information, and can handle uniform grids with missing data as well as nonuniform grids or random sampling.
\end{abstract}

The output grid of the 1D interpolation in the first step is arbitrary. When the output grid has uniform inline midpoints spacing, the $3 \mathrm{D}$ Fourier reconstruction in the second step is performed in the crossline midpoint, absolute offset, and azimuth coordinates. When the first step outputs to uniform absolute offset, the 3D Fourier reconstruction handles the crossline/inline midpoint and the azimuth coordinates. In both cases, the main innovation is the inclusion of the azimuthal coordinate in the Fourier reconstruction. The azimuth multiplicity must be increased for the method to be successful, which means that overlap shooting is required. We have tested the algorithm on synthetic streamer data for which the proposed method outperforms an approach where the azimuthal coordinate is ignored. Potential applications are interpolation of marine streamer data to decrease the crossline source sampling for the benefit of 3D multiple prediction and regularization to reduce sampling-related differences in processing of time-lapse data.

\section{INTRODUCTION}

Interpolation of seismic data has received renewed attention over the last few years with the widespread use of advanced seismic processing algorithms and the increased use of time-lapse seismics. For instance, 3D surface-related multiple elimination (SRME) requires data that are sampled uniformly and densely in the four spatial coordinates of a 3D seismic survey (two coordinates for the sources and two for the receivers). Sampling of 3D seismic data is rarely dense enough to meet the requirements of 3D SRME, and among the solutions to this problem is one whereby a densely sampled volume is reconstructed prior to multiple prediction (Hokstad and Sollie, 2004,
Baumstein, 2004). Another example is wave-equation migration, which also requires densely and uniformly sampled seismic data. Liu et al. (2003) discuss how interpolation prior to migration benefits the migration result. For time-lapse processing, the importance of source-receiver position repeatability can be hardly overestimated. Where such repeatability is lacking, regularization methods can help (Eiken et al., 2003).

\section{Seismic data regularization methods}

In seismic processing, data usually are regularized using transformation-based methods, filter-based methods, or wavefield-operator-

Manuscript received by the Editor August 26, 2005; revised manuscript received February 22, 2006; published online October 31, 2006.

${ }^{1}$ Formerly Delft University of Technology, Department of Applied Physics, Delft, The Netherlands; presently Shell International Exploration and Production, Kessler Park 1, 2288 GS, Rijswijk, The Netherlands. E-mail: paul.zwartjes@ shell.com.

${ }^{2}$ Delft University of Technology, Department of Applied Physics, P. O. Box 5046, Lorentzweg 1, 2600 GA, Delft, The Netherlands. E-mail: a.gisolf@ tnw.tudelft.nl.

(C) 2006 Society of Exploration Geophysicists. All rights reserved. 
based methods. Transformation-based reconstruction requires estimation of the coefficients of a particular transform (Fourier, Radon, wavelet, etc.) that synthesize the signal on the nonuniform grid, either through inversion (Hugonnet and Canadas, 1997; Duijndam et al., 1999b; Trad et al., 2003) or through an iterative approach (Kabir and Verschuur, 1995; Xu et al., 2005). These methods are fast when the transform can be computed efficiently, require little user input, and can handle both nonuniform and uniform grids. In a time-domain implementation of this approach, one scans along a number of trajectories, such as lines or parabolic/hyperbolic curves, to identify the main events, which are then interpolated to a finer grid (Bardan, 1987).

Filter-based methods interpolate by convolution with an interpolating filter in the spatial domain. A common method in seismic data processing for interpolation of uniformly sampled aliased data is prediction-error filters (Spitz, 1991). Fomel (2000) discusses how to use prediction-error filters on uniformly sampled data sets with gaps. These methods are not suited for general nonuniform sampling.

Wavefield reconstruction methods based on dip moveout (DMO) or migration operators and their transposes or least-squares inverses offer a theoretically attractive framework to seismic data reconstruction because they explicitly consider wave propagation. Artifacts resulting from nonuniform sampling can be reduced by trace weighting (Canning and Gardner, 1998) or adopting an inversion approach (Ronen, 1987; Nemeth et al., 1999). Biondi et al. (1998) chained the DMO and inverse DMO operators into a single operation called azimuth moveout, or AMO. A positive feature of wavefield-operator-based reconstruction methods is that they allow maximum use of available subsurface information. However, this strength also can be a weakness if the subsurface information is unknown or erroneous. Wavefield-continuation methods typically are computationally expensive.

\section{Fourier reconstruction}

The main reason for investigating Fourier reconstruction, one of the transformation-based reconstruction methods, is that usually the data are not sampled on a regular grid, and we want to avoid binning that introduces errors, especially for time-lapse processing. Also, we want to interpolate without explicitly specifying knowledge of the subsurface. The Fourier reconstruction approach is computationally efficient, requires no subsurface information, and can handle uniform grids with missing data as well as nonuniform grids or random sampling (Duijndam et al., 1999b; Hindriks and Duijndam, 2000).

The Fourier coefficients that describe the nonuniformly sampled data are estimated via a least-squares inversion. By incorporating a nonquadratic penalty function in the inversion, the reconstruction in gaps is improved (Zwartjes and Duijndam, 2000). Also, nonuniformly sampled, aliased seismic data can be reconstructed (Zwartjes and Sacchi, 2004). We call this method Fourier reconstruction with sparse inversion (FRSI). The method is currently limited to two spatial dimensions, and the main contribution of this paper is the extension to three spatial dimensions via the inclusion of the azimuthal coordinate. A similar algorithm has been published by Liu and Sacchi (2004) under the name minimum-weighted norm inversion.
Liu et al. (2004b) discuss how this algorithm can be used to reconstruct uniformly sampled land data in four spatial coordinates with gaps attributable to misfires, dead traces, and widely spaced source and receiver lines. The main difference of our method is that it can handle nonuniformly sampled data and uses a more general nonquadratic model penalty in the inversion.

\section{Regularization of streamer data using 10 sinc interpolation and 2D Fourier reconstruction}

Schonewille et al. (2003) proposed a two-step reconstruction scheme for 3D marine-streamer data. In the first step, a 1D sinc interpolation is used to reposition the hydrophones on the feathered streamer to a uniform sampling interval (Koek and Ongkiehong, 1997). The output coordinates are piecewise, linearly interpolated from the input coordinates, thereby accommodating deviations from a straight line. The output grid is arbitrary and can be chosen, for instance, as a grid with uniform inline offset, inline midpoint or absolute offset spacing. In Schonewille et al. (2003), the output grid is chosen to have uniform inline midpoints spacing. Nonuniform sampling then remains in the crossline midpoint, absolute offset, and azimuthal coordinates. In the second step, the reconstruction problem is reduced from three spatial dimensions to two by ignoring the azimuth coordinate. This assumption is based on the observation that at far offsets, the data are approximately single azimuth; at near offsets, the azimuthal variation in the data is often negligible. The data are reconstructed in the $2 \mathrm{D}$ crossline midpoint versus offset domain, optionally using normal moveout (NMO) to reduce the spatial bandwidth.

Schonewille (2003) presents a modification to this scheme to handle azimuthal traveltime variations resulting from dipping layers. The modification exists because the second step is no longer performed using 2D Fourier reconstruction but instead uses a modified 2D linear Radon transform that includes dip-dependent traveltime effects. This includes azimuthal traveltime effects implicitly in the modeling, but this approach cannot model other azimuthal amplitude/traveltime variations, such as those caused by azimuthal anisotropy. The resulting algorithm showed improved reconstruction of seismic data with azimuthal traveltime effects caused by dipping layers.

\section{Regularization of streamer data using 1D sinc interpolation and 3D Fourier reconstruction}

The approach by Schonewille (2003) does not describe amplitude variations with azimuth or traveltime variations caused by anisotropy. Therefore, we have implemented the Fourier reconstruction with sparse inversion approach in three spatial dimensions to incorporate explicitly the azimuthal coordinate [an extension of the nonuniform fast Fourier transform (NFFT) theory to $L$ dimensions is discussed in Duijndam and Schonewille, 1999a]. We have extended the model space with a full dimension by including the azimuthal wavenumber, although the amount of data on input remains the same. The extra information required to solve the now underdetermined inversion is provided by a sparseness constraint on the Fourier coefficients. 
Our procedure constitutes a reconstruction scheme for all four spatial coordinates in marine-streamer data, and we will compare this with the approach of Schonewille et al. (2003), which ignores the azimuthal coordinate. In the first step we will interpolate the data along streamers to uniform inline midpoint or absolute offset coordinates. In the second step, we will then handle the nonuniform sampling in the remaining three spatial coordinates, using the 3D FRSI algorithm. This procedure constitutes a reconstruction scheme for all four spatial coordinates.

The objective of this paper is to demonstrate the feasibility of this approach. Although we use a Fourier transformation for the reconstruction, one could use the same concept with the linear Radon transform.

In the next section, we discuss the inversion for the Fourier coefficients. The requirements on the sampling in the azimuthal coordinate are discussed as well as expected traveltime variation with azimuth resulting from dipping layers. We demonstrate the feasibility of the method on two synthetic examples. In the first example, the seismic data shows azimuthal traveltime variation attributable to anisotropy; in the second example, the variation is caused by the presence of dipping layers. We conclude the paper with a brief summary of key aspects of our method.

\section{D FOURIER RECONSTRUCTION}

In Fourier reconstruction, the seismic data are reconstructed per temporal frequency. At each frequency $f$, the Fourier coefficients $\widetilde{\mathbf{p}}$ are estimated from the nonuniformly sampled data $\mathbf{p}$. This is done via least-squares inversion of the inverse Fourier transform matrix, which is defined in equation A-2 and denoted by A. Noise and other sources of error, such as coherent energy outside the specified bandwidth, are swept together into a single term $\mathbf{n}$. The solution to the linear system $\mathbf{p}=\mathbf{A} \widetilde{\mathbf{p}}+\mathbf{n}$ is defined as the minimum of an objective function with a quadratic penalty on the data fit and a nonquadratic penalty on the model term,

$$
J=\frac{1}{\sigma_{n}^{2}}\left\|\mathbf{W} \frac{1}{2}(\mathbf{A} \widetilde{\mathbf{p}}-\mathbf{p})\right\|_{2}^{2}+\rho(\widetilde{p}) .
$$

Table 1. Penalty functions, $\rho(x)$ and corresponding influence function $\psi(x)$

Matrix $\mathbf{W}$ is a diagonal matrix whose terms denote the weights given to individual traces. In the following examples, we have set $\mathbf{W}=\mathbf{I}$. The scalar $\sigma_{n}^{2}$ denotes the a priori noise variance, but in practice is used as a tuning parameter. The model penalty function that we use is defined as in Zwartjes (2005):

$$
\rho\left(\tilde{p}_{i} ; a, \sigma_{\tilde{p}}\right)=\frac{1}{2(1-a)}\left(\tilde{p}_{i} \tilde{p}_{i}^{*}+\sigma_{\widetilde{p}}^{2}\right)^{1-a} .
$$

It has tuning parameters $a$ and $\sigma_{\tilde{p}}^{2}$ to determine the weight given to individual model parameters, which can be thought of as sparseness tuning parameters. An estimator for the minimum of the objective function defined in equation 1 is

$$
\hat{\widetilde{\mathbf{p}}}=\left(\mathbf{A}^{H} \mathbf{W} \mathbf{A}+\sigma_{n}^{2} \mathbf{C}_{\tilde{p}}\right)^{-1} \mathbf{A}^{H} \mathbf{W} \mathbf{p},
$$

where the model weight matrix $\mathbf{C}_{\tilde{p}}$ is diagonal with terms given by

$$
C_{\widetilde{p}, i i}^{-1}=\frac{1}{\widetilde{p}_{i}} \frac{\partial \rho\left(\tilde{p}_{j}\right)}{\partial \widetilde{p}_{i}}=\left(\widetilde{p}_{i} \widetilde{p}_{i}^{*}+\sigma_{\widetilde{p}}^{2}\right)^{-a} .
$$

We call this a Fourier reconstruction with sparse inversion (Zwartjes, 2005). Some common model penalty functions $\rho(\widetilde{p})$ and associated expressions for the diagonal terms of the model weight matrices $C_{\tilde{p}, i i}^{-1}$ are listed in Table 1 . For $a=1 / 2$ in equation 2 , the weight matrix $\mathbf{C}_{\tilde{p}}$ is similar to the model weight matrix obtained with an $\ell_{1_{-2}}$ norm model penalty (as an $\ell_{1_{-2}}$ norm, but with a smooth first derivative). And for $a=1$, it is similar to the estimator obtained with a Cauchy penalty function (Sacchi and Ulrych, 1995). Equation 4 was inspired by the relation between the diagonal terms of the model weighting matrices obtained using the $\ell_{1_{-}}$, Cauchy and Geman-McClure penalty functions. A scale parameter $\sigma_{\tilde{p}}^{2}$ has been added to allow some tuning of the amount of sparseness. For a given $\sigma_{\tilde{p}}^{2}$, a value of $a=1$ yields a sparser result than $a=1 / 2$. The matrix $\mathbf{C}_{\tilde{p}}$ can be smoothed to obtain a sparse but not spiky solution from the sparse inversion (Liu and Sacchi, 2004).

\section{Efficiency}

The main computational effort in equation 3 is in the evaluation of the factor $\mathbf{A}^{H} \mathbf{W} \widetilde{\mathbf{p}}$ (the NDFT) and the inversion of the matrix $\mathbf{A}^{H} \mathbf{W A}$. Straightforward evaluation of the NDFT via a matrix-vector multiplication is prohibitively expensive; therefore, we have extended the NFFT algorithm by Duijndam et al. (1999b) to three spatial dimensions (see Appendices A and B). The inversion is solved efficiently with the conjugate gradient (CG) method because matrix $\mathbf{A}^{H} \mathbf{W A}$ is a 3D Toeplitz matrix. Additionally, we accelerate the CG

\begin{tabular}{|c|c|c|c|}
\hline Penalty function & $\rho(x)$ & $\psi(x)=\frac{\partial \rho(x)}{\partial x}$ & $C_{x}^{-1}=\frac{\psi(x)}{x}$ \\
\hline Quadratic $\left(\ell_{2}\right)$ & $\frac{1}{2} x^{2}$ & $x$ & 1 \\
\hline Absolute value $\left(\ell_{1}\right)$ & $|x|$ & $\operatorname{sgn}(x)$ & $\frac{1}{|x|}$ \\
\hline Huber $\left\{\begin{array}{l}|x| / c \leq \pi / 2 \\
|x| / c>\pi / 2\end{array}\right.$ & $\left\{\begin{array}{l}c^{2}(1-\cos (x / c) \\
c|x|+c^{2}(1-\pi / 2)\end{array}\right.$ & $\left\{\begin{array}{l}c \sin (x / c) \\
c \operatorname{sgn}(x)\end{array}\right.$ & $\left\{\begin{array}{l}\operatorname{sinc}(x / c) \\
\frac{c}{|x|}\end{array}\right.$ \\
\hline$\ell_{1-2}$ & $\sqrt{1+x^{2}}$ & $\frac{x}{\sqrt{1+x^{2}}}$ & $\left(1+x^{2}\right)^{-1 / 2}$ \\
\hline Cauchy & $\frac{1}{2} \ln \left(1+x^{2}\right)$ & $\frac{x}{1+x^{2}}$ & $\left(1+x^{2}\right)^{-1}$ \\
\hline Geman-McClure & $\frac{1}{2} \frac{x^{2}}{1+x^{2}}$ & $\frac{x}{\left(1+x^{2}\right)^{2}}$ & $\left(1+x^{2}\right)^{-2}$ \\
\hline Generalized function & $\frac{1}{2(1-a)}\left(x^{2}+1\right)^{1-a}$ & $x\left(1+x^{2}\right)^{-a}$ & $\left(1+x^{2}\right)^{-a}$ \\
\hline
\end{tabular}
scheme by preconditioning the matrix $\mathbf{A}^{H} \mathbf{W A}$ with the weighting matrix $\mathbf{C}_{\tilde{p}}$. Trad et al. (2003) use a similar algorithm for the highand penalty function $C_{x}^{-1}$ (equation 4$)$ 
resolution Radon transform. We refer to that paper for implementation details. Further efficiency, in particular for the inversions at lower temporal frequencies, is obtained by reducing the number of parameters or Fourier coefficients in the inversion based on the maximum dip in the data. This bandwidth restriction is not applied to the azimuthal wavenumber.

\section{Automatic determination of sparseness parameters}

A useful practical rule is to set $\sigma_{\tilde{p}}^{2}$ equal to a percentage or percentile of the maximum of the squared NDFT coefficients. However, estimating appropriate values for the penalty function parameters $a$
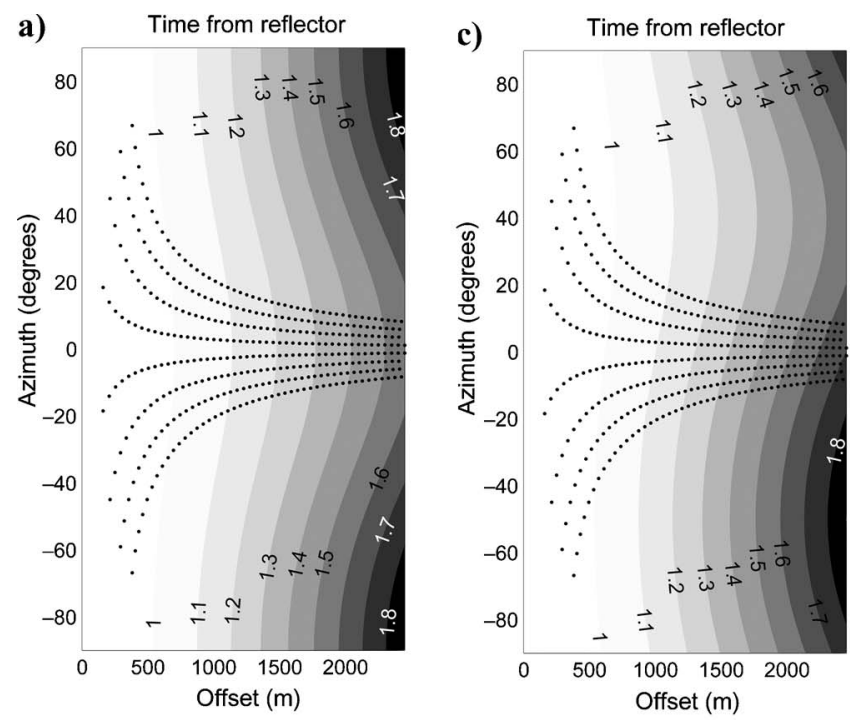

b) Difference from zero azimuth time
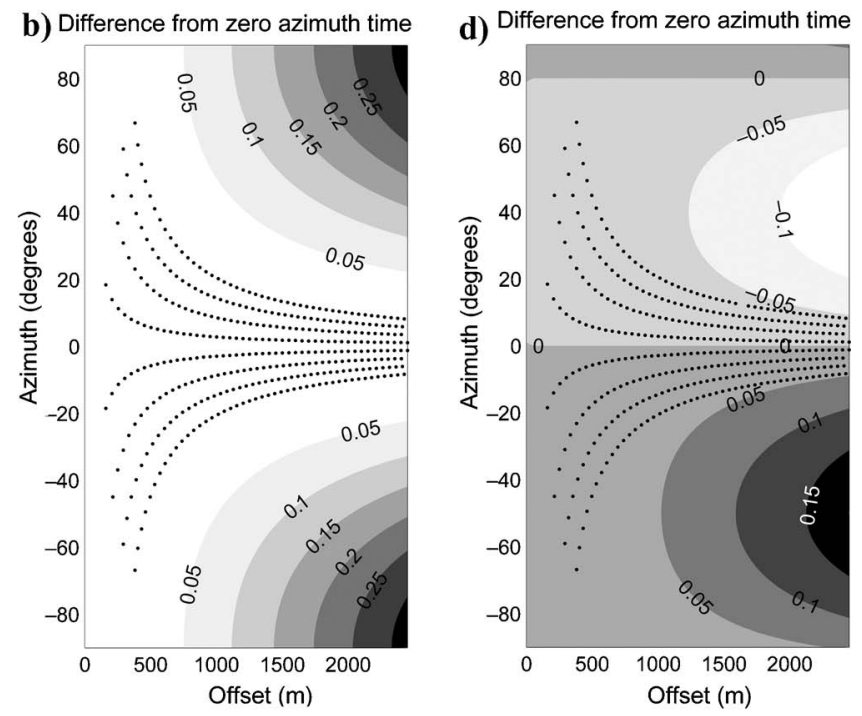

Figure 1. Reflection time (s) as a function of absolute offset and azimuth from a single dipping reflector at a 1400 -m depth with a velocity of $3000 \mathrm{~m} / \mathrm{s}$. Overlaid are the sampling positions of a typical marine streamer survey. The inline dip is $40^{\circ}$. (a) Traveltimes for a crossline dip of $0^{\circ}$ and (b) the corresponding difference with respect to zero-azimuth traveltime. (c) Traveltimes for a crossline dip of $40^{\circ}$ and (d) the corresponding difference with respect to zero-azimuth traveltime. and $\sigma_{\tilde{p}}^{2}$ via trial and error is prohibitively expensive for 3D FRSI. We estimate these parameters from the data by matching a generic penalty function against a histogram of Fourier coefficient amplitudes, which are obtained using the NDFT defined in equation A-1. We rewrite equation 2 as a distribution

$$
y\left(\widetilde{p}_{i} ; a, \sigma_{\tilde{p}}\right)=C e^{-\rho\left(\tilde{p}_{i} ; a, \sigma_{\tilde{p}}\right)},
$$

with $\rho\left(\widetilde{p}_{i} ; a, \sigma_{\tilde{p}}\right)$ as defined in equation 2 . The distribution $y\left(\widetilde{p}_{i} ; a, \sigma_{\tilde{p}}\right)$ depends on tuning parameters $a$ and $\sigma_{\tilde{p}}$, and the factor $C$ is a normalization constant. For a range of values of $a$ and $\sigma_{\tilde{p}}^{2}$, the fit to the histogram is computed, and the values yielding the best fit are used in the inversion. Because we aim to find useful parameters for the inversion, we fit the generic penalty function to the part of the histogram beyond one or two times the histogram's standard deviation to avoid fitting to the noise component.

\section{Sampling requirements for reconstruction in four spatial coordinates}

In marine-streamer acquisition, the shot and receiver coordinates in the inline direction usually are well sampled, whereas the coordinates in the crossline direction are not. The crossline shot-sampling interval is the most sparsely sampled coordinate, with a sampling interval usually equal to half the sail-line width, which easily can be several hundred meters. Standard practice is to have half-overlapping swaths that yield single coverage in the crossline-midpoint coordinate. This means that for each midpoint-offset location, there is only one azimuth, which we refer to as single-azimuth multiplicity. Seismic data cannot be interpolated or reconstructed in the azimuthal direction based on one trace only. To overcome this problem in the synthetic examples, we increased the azimuth multiplicity by sailing as if the boat towed fewer streamers than it actually did, yielding an overlap in midpoints. In the first example, we have eight streamers with $75 \%$ overlap in the swaths yielding an azimuth multiplicity of two. In the second example, we have ten streamers — but with a crossline roll-along as if there were eight — yielding an azimuth multiplicity of two only at the edges of a swath. To our knowledge, the first mode of acquisition has never been implemented. The acquisition pattern of the second example has been used by Widmaier et al. (2003) to minimize azimuthal differences in data processing between surveys in time-lapse experiments.

\section{Traveltime variation with azimuth}

When traveltime varies with azimuth, the moveout is periodic with an azimuth period of $180^{\circ}$ because of reciprocity. In marinestreamer surveys, the range in azimuths is usually so small that the azimuthal moveout is approximately linear. Linear azimuthal moveout can be described well with a few Fourier coefficients, provided the data are not aliased.

Figure 1 shows the traveltime variation resulting from a single dipping layer one can expect for a given midpoint (not reflection point) as a function of offset $(h)$ and azimuth $(\phi)$. This layer has a dip angle of $\alpha=40^{\circ}$, a midpoint-to-reflector distance of $d_{m}=1400 \mathrm{~m}$, 
and a velocity $v=3000 \mathrm{~m} / \mathrm{s}$. Figure $1 \mathrm{a}$ and $\mathrm{b}$ shows results for a dip direction $\theta=0^{\circ}$, and Figure $1 \mathrm{c}$ and $\mathrm{d}$ shows results for a dip direction of $\theta=40^{\circ}$. The traveltime values were computed using equation 1 from Chemingui and Baumstein (2000):

$$
t(h, \phi)=\frac{2}{v} \sqrt{d_{m}^{2}+h^{2}\left(1-\sin \alpha^{2} \cos ^{2}(\phi-\theta)\right.} .
$$

as if it carried only four streamers instead of eight. This type of acquisition would be called as double overlap shooting, based on the fact that each midpoint and offset is sampled twice with different azimuths.

To achieve good results with the 3D FRSI algorithm, the reconstruction in the following example will be performed in subsets of limited-offset range of the full $\left(m_{y},|h|, \phi\right)$ domain.
Figure 1b shows that for a zero dip direction and $40^{\circ}$ dip angle, the traveltime difference from zero azimuth at the near offsets is about the same as at the large offsets and is less than 10-20 ms. Figure $1 \mathrm{~d}$ shows that, in the case of a $40^{\circ}$ dip direction, timing differences between positive and negative offsets can be as large as $100 \mathrm{~ms}$. Also, the azimuthal moveout within the range acquired by streamer data is approximately linear (Figure 1c).

\section{Reconstruction in the common inline midpoint domain}

As discussed in the introduction, the output grid of the 1D interpolation in the first step is arbitrary and can be chosen, for instance, as uniform inline midpoint or uniform absolute-offset sampling. In the original paper by Schonewille et al. (2003), a choice is made for uniform inline midpoint sampling. This leaves nonuniform sampling in the crossline midpoint $\left(m_{y}\right)$, absolute offset $(|h|)$, and azimuth $(\phi)$ coordinates. An advantage of this approach is that the spatial bandwidth along the offset coordinate can be reduced by application of NMO, thereby reducing the number of significant parameters in the inversion. The bandwidth in the crossline midpoint direction depends on the geology. Here we follow that same approach, i.e., the data are interpolated along the streamers to uniform inline midpoint sampling, and the 3D Fourier reconstruction is performed in a common inline midpoint volume.

The sampling geometry along the $\left(m_{y},|h|, \phi\right)$ coordinates for a single-source, eight-streamer survey with four parallel swaths is shown in Figure 2a-2d. The swaths are half-overlapping, which yields a single-azimuth multiplicity or one azimuth per crossline midpoint, as can be seen from Figure $2 b$. This acquisition style is also termed no-overlap shooting, based on the fact that each midpoint/offset combination is sampled once. We cannot interpolate in the azimuthal direction based on one trace only; therefore, we double the azimuth multiplicity to test the feasibility of our algorithm. Figure $2 \mathrm{e}-\mathrm{h}$ shows the acquisition geometry for a doubling in azimuth multiplicity, which yields two traces with different azimuths at each midpoint/offset location. To achieve an azimuth multiplicity of two, adjacent swaths must overlap by $75 \%$. This can be achieved by sailing the boat conventionally, but
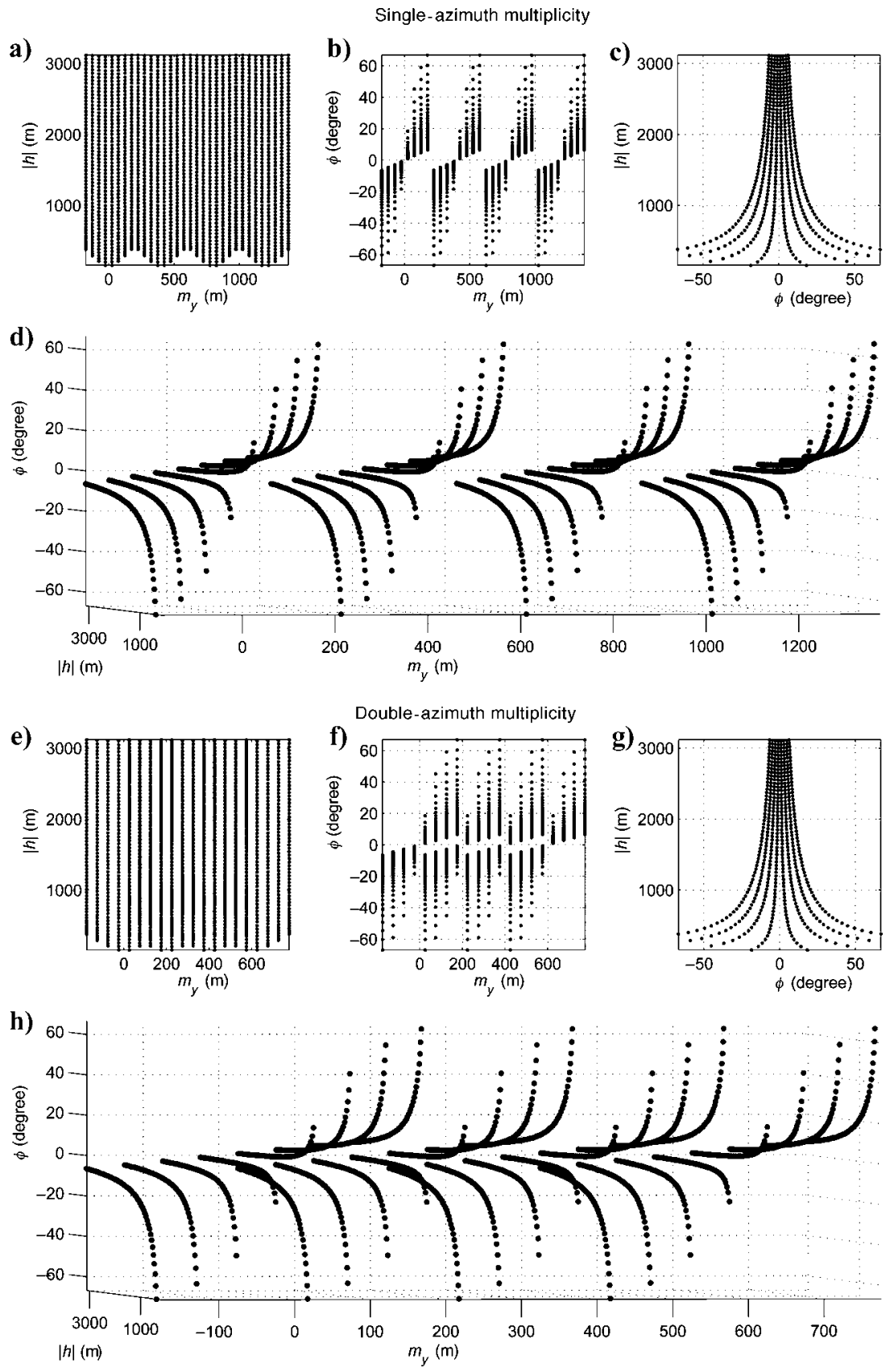

Figure 2. (a-c) Sampling in crossline midpoint $\left(m_{y}\right)$, absolute offset $(|h|)$, and azimuth $(\phi)$ coordinates for a single-source and eight-streamer survey with azimuth multiplicity of one shown in subsets of two coordinates and (d) shown for all coordinates in a 3D plot. (e-g) Sampling in crossline midpoint $\left(m_{y}\right)$, absolute offset $(|h|)$, and azimuth $(\phi)$ coordinates, but now for an azimuth multiplicity of two, shown in subsets of two coordinates and $(\mathrm{h})$ for all coordinates in a $3 \mathrm{D}$ plot 


\section{Synthetic example with $\boldsymbol{P}$-wave anisotropy}

Synthetic data were generated for a horizontally layered model with an elliptical, azimuthal anisotropy. The anisotropy ellipse makes an angle of $45^{\circ}$ with the inline direction for all layers so that the positive azimuths are faster than the negative azimuths. The synthetic survey consists of six swaths with eight streamers, and all swaths were acquired in the same direction. The azimuth multiplicity was set to two by having $75 \%$ overlap between swaths. The maximum offset is $3150 \mathrm{~m}$ with a near-offset gap of $150 \mathrm{~m}$ and a $100-\mathrm{m}$ streamer separation. The shot interval was $25 \mathrm{~m}$ with $4-\mathrm{m}$ random source/receiver positioning error and $25-\mathrm{m}$ cable snaking. No noise was added.

\section{Reconstruction settings}

Reconstruction results are shown for a far-offset volume (2000-3000 m). The input data consisted of 1303 traces in 31 CMPs. NMO with a constant velocity of $2000 \mathrm{~m} / \mathrm{s}$ was applied to re duce the spatial bandwidth. We invert for 11 Fourier coefficients along the crossline wavenumber direction, symmetric around $k_{m_{y}}$ $=0$, and 21 in the offset wavenumber direction ( 15 for downward and five for upward curvature). All events are horizontal in the crossline midpoint axis and almost horizontal in the offset axis because of the NMO correction. Therefore, most energy is expected around $\left(k_{m_{i}}, k_{h}\right)=0$. Because of the anisotropy, the events also show quite significant traveltime variation with azimuth (see Figure 6). We have chosen 11 Fourier coefficients, symmetric around $k_{\phi}=0$. The sampling interval $\Delta k$ is based on the aperture in the corresponding spatial dimension, i.e., $\Delta k_{x}=2 \pi /\left(x_{\max }-x_{\min }\right)$.

The spatial bandwidth in the inversion is made frequency dependent by a minimum apparent velocity in the crossline midpoint and offset coordinates of $2500 \mathrm{~m} / \mathrm{s}$. The maximum frequency used in the inversion is $60 \mathrm{~Hz}$. These settings yield a maximum number of 2541 model parameters, which is almost twice the number of input traces. Even within this velocity cone, the number of parameters already exceeds the number of traces at a frequency of $12 \mathrm{~Hz}$. The assumption of model sparseness provides the prior information required to solve this underdetermined inversion.
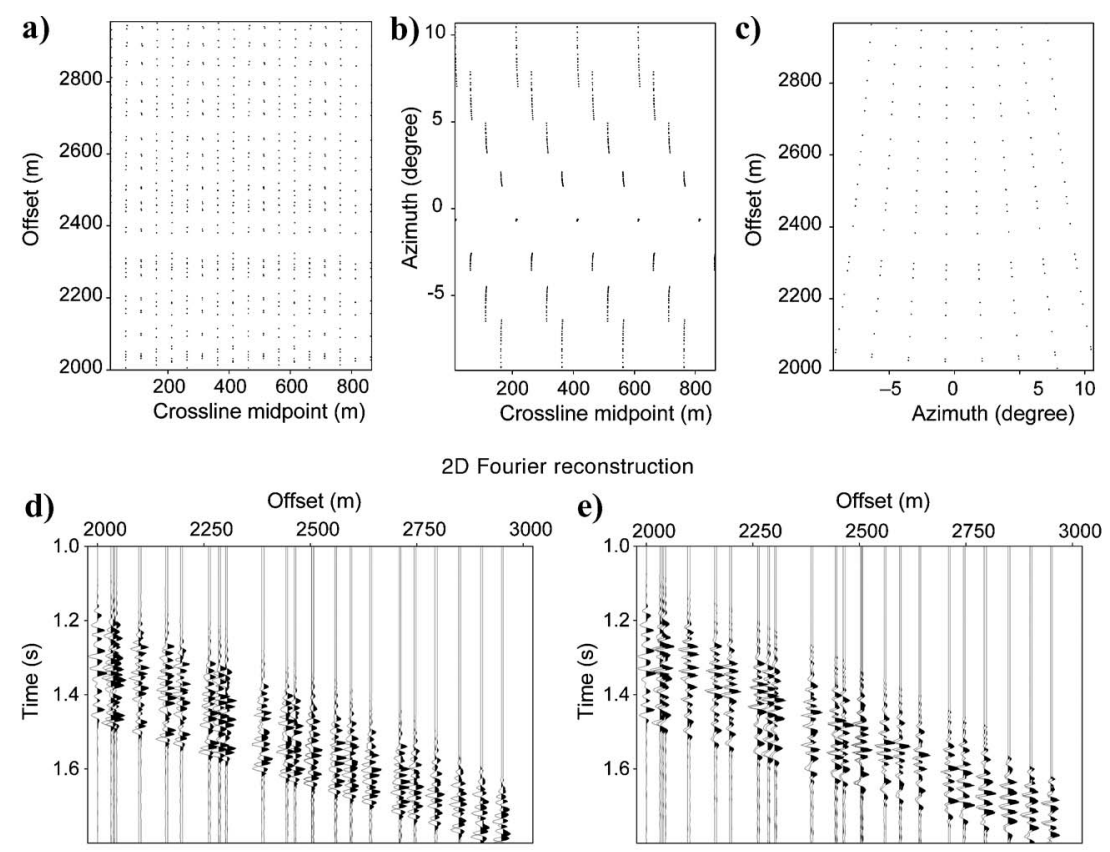

e)
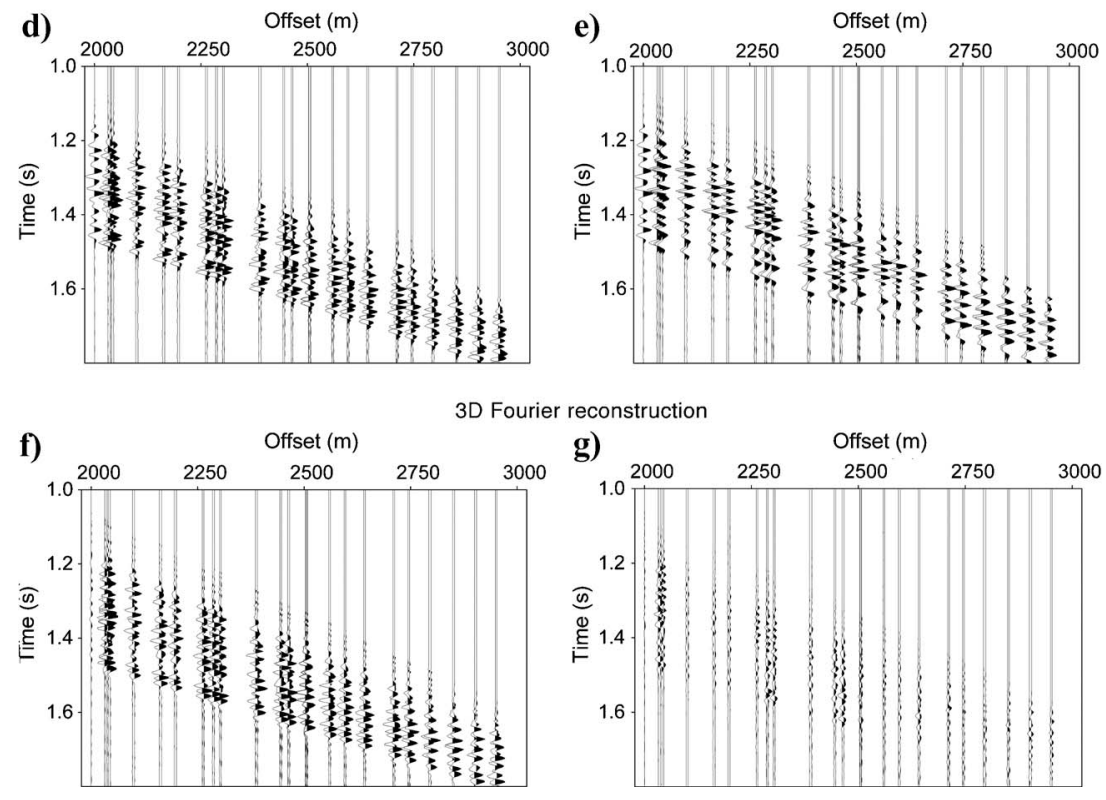

Figure 3. Sampling geometry for offsets between 2000 and $3000 \mathrm{~m}$ (a) Crossline midpoint versus offset; (b) crossline midpoint versus azimuth; (c) azimuth versus offset coordinates; (d) 2D FRSI reconstruction to the original grid; (e) difference with the input data; (f) 3D FRSI reconstruction to the original grid; and (g) difference with the input data.

\section{Reconstruction results}

As a means of reconstruction quality control, the data can be reconstructed to the input coordinates to inspect the difference with the input data. Figure 3 shows a common-midpoint gather extracted from the $2 \mathrm{D}$ and $3 \mathrm{D}$ FRSI reconstructions to the original grid. Because of the anisotropy, the reflection events are not perfect hyperbolas but show some jitter. The 2D FRSI algorithm neglects the azimuth and has problems to reconstruct properly the azimuthal variation if the difference in offset between the two traces is small. Figure 3d shows how 2D FRSI averages the azimuthal variation. The 3D FRSI algorithm reconstructs the azimuthal effect much better (Figure $3 \mathrm{f}$ and $\mathrm{g}$ ). Figure $4 \mathrm{a}$ and $\mathrm{b}$ shows the same common-midpoint gathers reconstructed to a regular grid with the 3D FRSI output to zero azimuth. Only the 3D FRSI algorithm yields a correct reconstruction.

In the absence of anisotropy, a common-offset section gives a clear representation of the subsurface. However, time shifts resulting from anisotropy cause jitter, as shown in Figure $4 \mathrm{c}$ for a common offset of $2275 \mathrm{~m}$. The modeled zero-azimuth common-offset gather at the same offset is shown in Figure 4f. The 2D FRSI reconstruction in Figure 4d averages the timing differences, which cancel for the shallow events and yields a result that resembles the true zero-azimuth data quite poorly. The 3D FRSI reconstruction in Figure $4 \mathrm{e}$ closely resembles the modeled data because it accommodates the azimuthal variation.

The 3D FRSI algorithm also can output data to a common azimuth different from zero, provided the chosen azimuth lies within the range of azimuths contained in the data. Figure 5 shows gathers from the 3D FRSI reconstruction at a common 
offset of $2275 \mathrm{~m}$ and a common azimuth of $+5^{\circ}$ and $-5^{\circ}$. The reconstruction closely matches the data modeled at these azimuths. Note that the positive azimuths are faster than the negative azimuths.

Figure 6a shows the variation with azimuth in the input data (for two CMPs) between $+10^{\circ}$ and $-10^{\circ}$ at $2275 \mathrm{~m}$ offset. Figure $6 \mathrm{~b}$ shows the 3D FRSI reconstruction results, which closely match the modeled data shown in Figure 6c. This also shows that for the limited range of azimuths acquired in streamer data, the moveout is approximately linear, although the azimuthal variation is periodic and shows a sinusoidal variation over the full range of $180^{\circ}$.

Note that the anisotropy in this example is quite strong and is rarely, if ever, observed on marine-streamer data. Milder anisotropy will result in less extreme traveltime variations. When the 2D Fourier reconstruction algorithm is applied to data acquired by a conventional single-azimuth multiplicity survey, the reconstruction typically will be much better, albeit with discontinuities and the sail-line boundaries. The next example shows a more realistic setting with overlap shooting that yields double-azimuth multiplicity only at the swath boundaries and azimuthal traveltime variation resulting from dipping structure. Here the differences between the 2D and 3D Fourier reconstruction algorithm are much smaller and become clearer only in a time-lapse experiment.

\section{Reconstruction in the} common-offset domain

In this example, the reconstruction is performed in the common-offset domain, which means the first step is to interpolate the data along the streamers to uniformly spaced absolute offsets. After this step, nonuniform sampling remains in the inline midpoint $\left(m_{x}\right)$, crossline midpoint $\left(m_{y}\right)$, and azimuth $(\phi)$ coordinates. An advantage of performing the reconstruction in common-offset volumes is that sampling irregularities resulting from feathering are reduced because feathering causes mainly an offset-dependent deviation from the uniform grid. By working in the common-offset domain, the effect of feathering on sampling positions is less severe. A disadvantage of working in common-offset volumes in respect to the inversion for the Fourier coefficients is that NMO no longer can be used to reduce the spatial bandwidth and aliasing artifacts. This is, in part, compensated by the fact that the sampling intervals in the midpoint domain are usually smaller than along the offset axis.

The ideal uniform-sampling geometry in the $\left(m_{x}, m_{y}, \phi\right)$ domain for a single-source, ten- a)

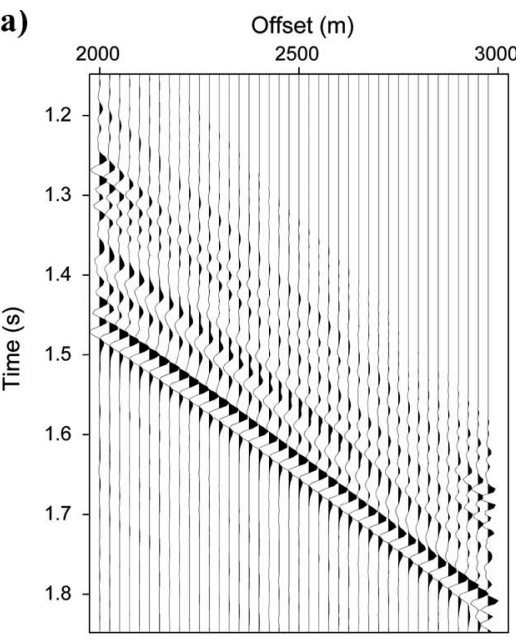

c)

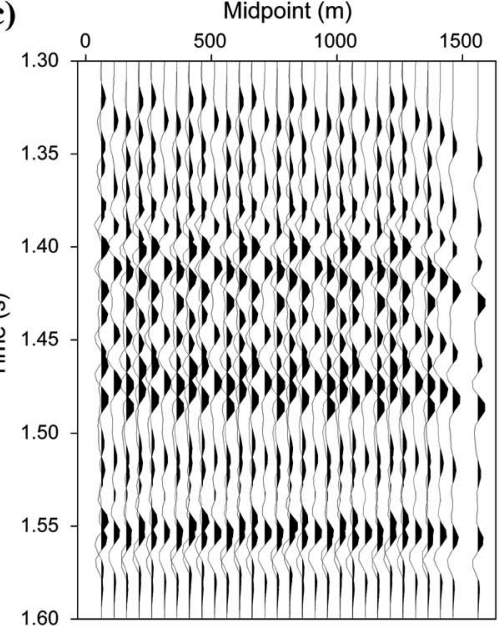

e)

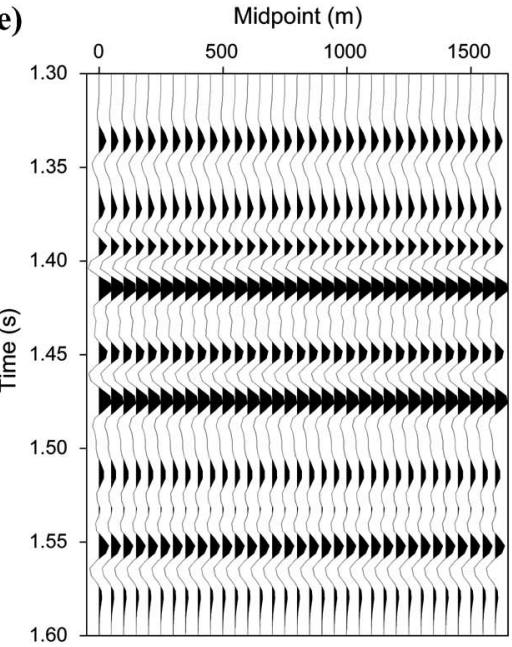

b)

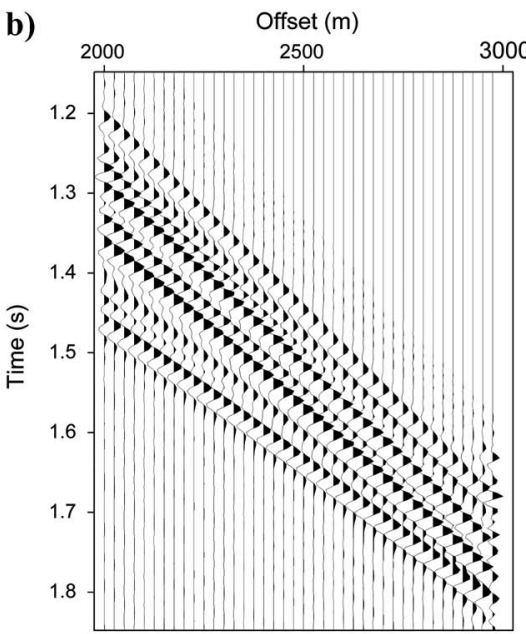

d)

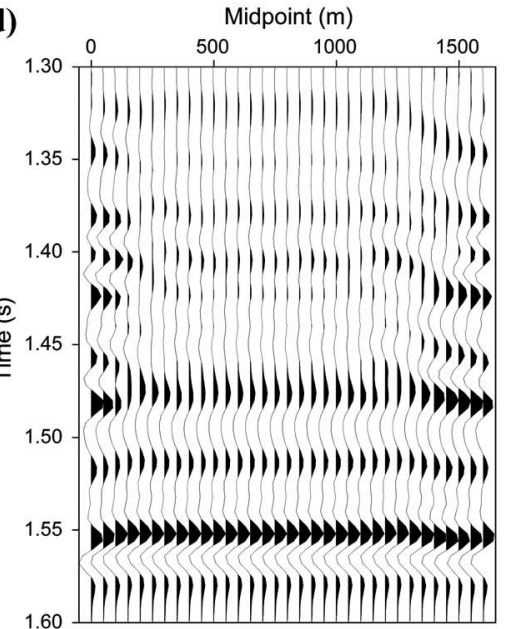

f)

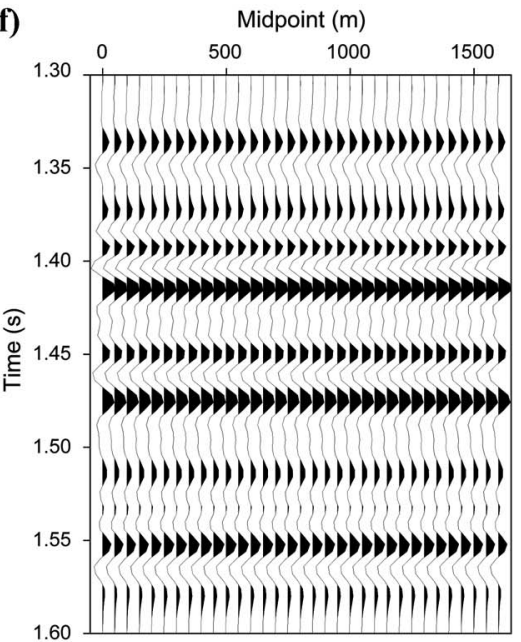

Figure 4. The common-midpoint gather of Figure 3 reconstructed to a uniform grid with (a) 2D FRSI and (b) 3D FRSI. The 2D FRSI algorithm neglects the azimuthal coordinate, while the 3D FRSI algorithm reconstructs to zero azimuth. (c) Common-offset gather at $2275 \mathrm{~m}$; (d) 2D FRSI reconstruction; (e) 3D FRSI reconstruction to zero azimuth; (f) data modeled at zero azimuth. 


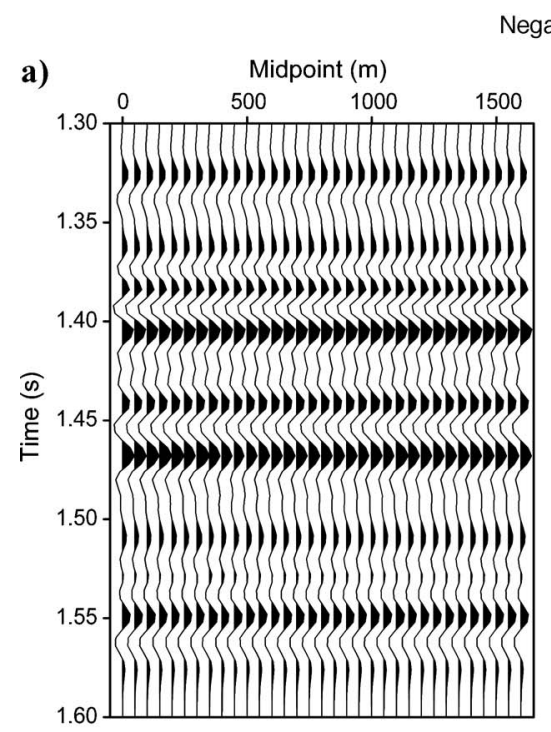

Negative azimuth

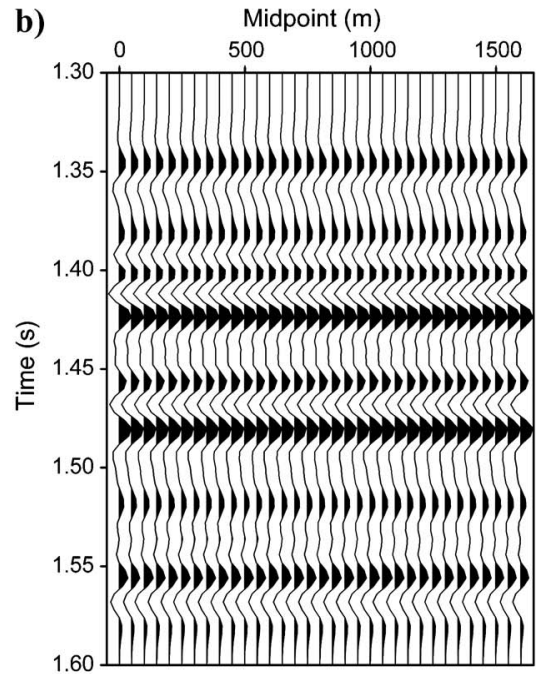

Positive azimuth

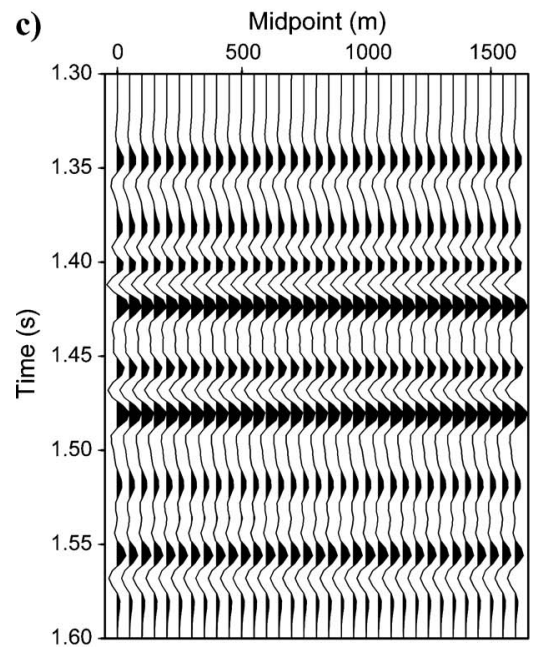

d)

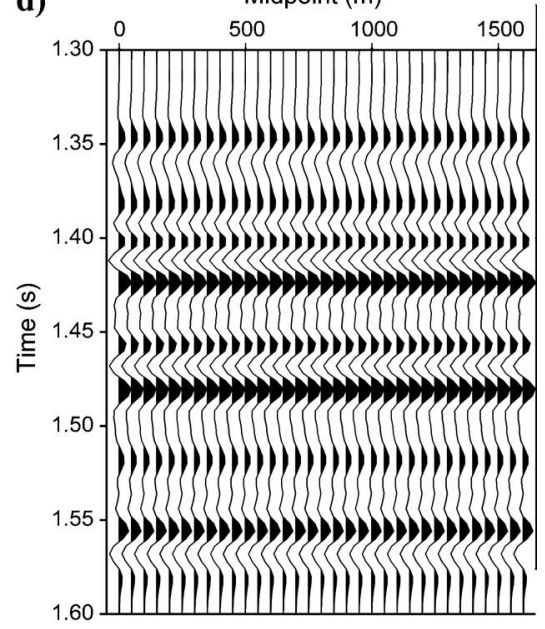

Figure 5. Common-offset gathers at $2275 \mathrm{~m}$ : (a) 3D FRSI reconstruction to an azimuth of $+5^{\circ}$ (b) the modeled data at that azimuth; (c) the modeled data at an azimuth of $-5^{\circ}$; (d) 3 D FRSI reconstruction at the azimuth.
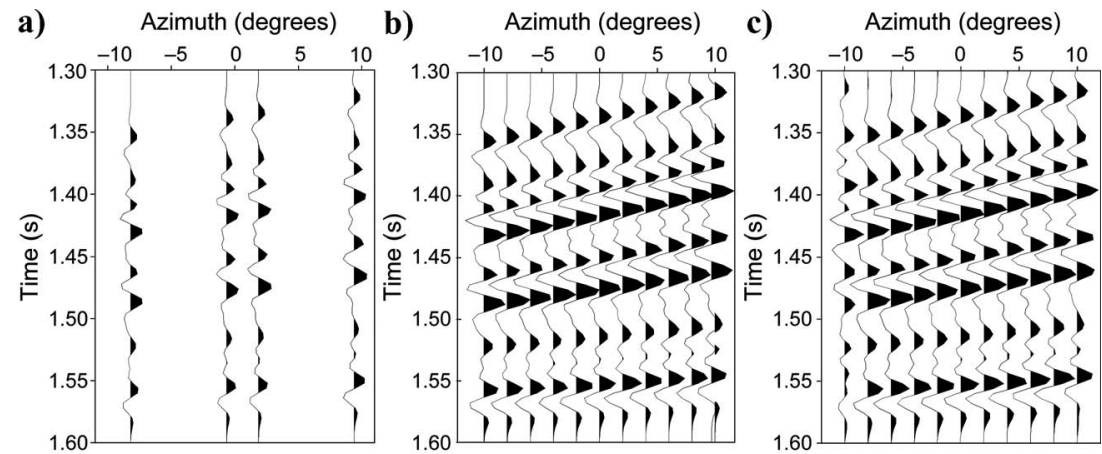

Figure 6. Common-midpoint, common-offset gathers showing azimuthal variation in the data: (a) input data at approximately $2275 \mathrm{~m}$ offset for two adjacent common midpoints; (b) 3D FRSI reconstruction at $2275 \mathrm{~m}$ offset for a single common midpoint; (c) modeled data at $2275 \mathrm{~m}$ offset for a single common midpoint. 
streamer marine-streamer survey is shown in Figure 7a-7d. In this example, the traveltime variation with azimuth is weaker, and we can relax the requirements on the azimuth multiplicity from a value of two, as in the previous example, to a value of two only at the swath boundaries, which is achieved by sailing as if the boat towed eight streamers instead of ten. This gives the 3D FRSI algorithm more data to handle the abrupt change in source-receiver azimuth going from the midpoints of one swath to those of the next. Figure 7e-h shows the sampling pattern in a common-offset volume resulting from this overlap shooting.

\section{Synthetic time-lapse example with a model with dipping layers}

In case of dipping layers, there will be a traveltime variation with both midpoint coordinates as well as with azimuth. The azimuthal traveltime effect caused by dipping layers is more subtle because of the limited range of azimuths covered by streamers (see Figure 13). However, in timelapse seismic processing, such subtle differences can be quite important. To test the performance of the 3D FRSI method in a time-lapse synthetic experiment, we generated two synthetic data sets using a simple model with reflectors dipping in both the inline and crossline directions. The data consist only of primary, acoustic reflections. Because nothing changed in the modeled subsurface or in the seismic wavelet, any difference after regularization results from the regularization method.

The marine-acquisition geometry consists of six parallel swaths, each swath consisting of ten streamers and overlapping the adjacent one. Figure $9 \mathrm{e}$ and fshows the overlap in azimuths at the edges of the swaths. The synthetic survey was modeled with a $12.5-\mathrm{m}$ inline receiver interval, 50-m crossline receiver (streamer) interval, 25-m inline shot interval, 175-m crossline shot (swath) interval, and Ricker wavelet as a source wavelet. Random noise with a signal-to-noise $(\mathrm{S} / \mathrm{N})$ ratio of about $6 \%$ was added. Small source and cablepositioning errors of a maximum $4 \mathrm{~m}$ and a combination of 25- $\mathrm{m}$ cable snaking and a varying parabolic feathering with maximum deviation of $150 \mathrm{~m}$ at $3000 \mathrm{~m}$ offset resulted in sampling differences between the two surveys.

Figure 8 shows the resulting nonuniform sampling geometry in a common-offset volume at $2000 \mathrm{~m}$ that was selected for this reconstruction experiment. The data consist of 50 inline CMPs with a range of $300 \mathrm{~m}$ at roughly $6.25 \mathrm{~m}$ sampling and 50 crossline CMPs with a midpoint range of $1250 \mathrm{~m}$ at about $25 \mathrm{~m}$ sampling and azimuths between $-5^{\circ}$ and $+10^{\circ}$. Both data sets contain approximately 780 traces.

Timing differences caused by dipping layers are noticeable in the common-offset data on both inline and crossline midpoint gathers. The azimuthal effect is apparent as a jitter along the re- flectors on the common crossline midpoint gathers in Figure $9 \mathrm{c}$ and $\mathrm{d}$ because adjacent traces have opposite azimuths as shown by the coordinates in Figure 9a and b. The jitter effect on the crossline midpoint gather in Figure 9d is subtler because adjacent traces have almost the same midpoint and all timing differences are purely attributable to differences in azimuth, which are relatively small.

On common inline midpoint gathers in Figure $9 \mathrm{~g}$ and $\mathrm{h}$, the reflectors appear stepwise continuous because of the discontinuity in azi-
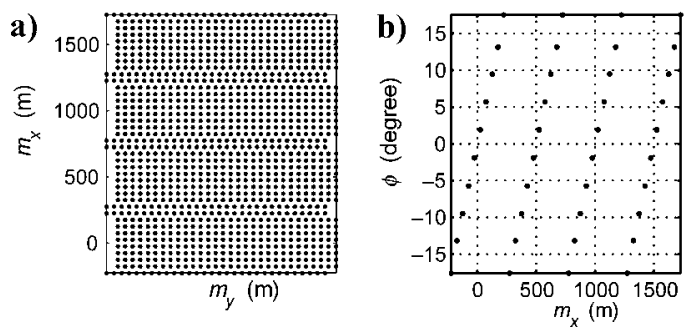
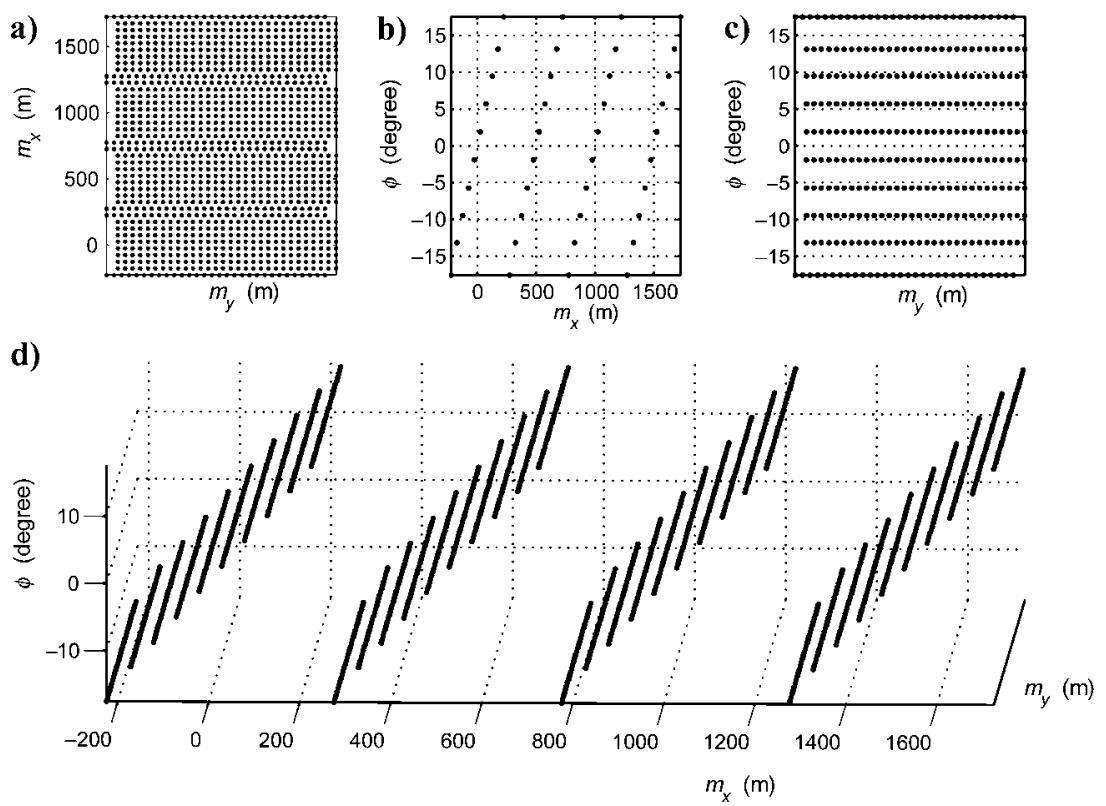

Double-azimuth multiplicity
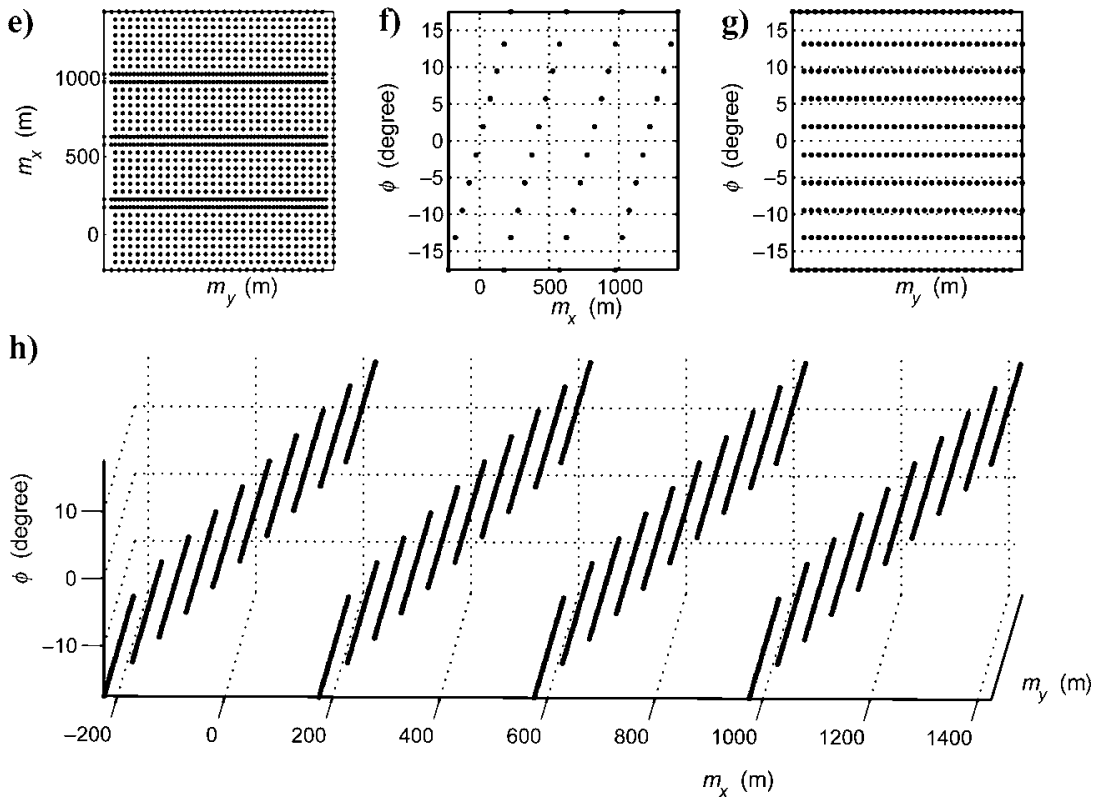

Figure 7. (a-c) Sampling in inline/crossline midpoint $\left(m_{x}, m_{y}\right)$ and azimuth $(\phi)$ coordinates for a single source and ten streamers survey with azimuth multiplicity of one shown in subsets of two coordinates and (d) shown for all coordinates in a 3D plot. (e-g) Sampling in $\left(m_{x}, m_{y}, \phi\right)$ coordinates for an azimuth multiplicity of two only at the edge of a swath, shown in subsets of two coordinates and (h) for all coordinates in a 3D plot. 

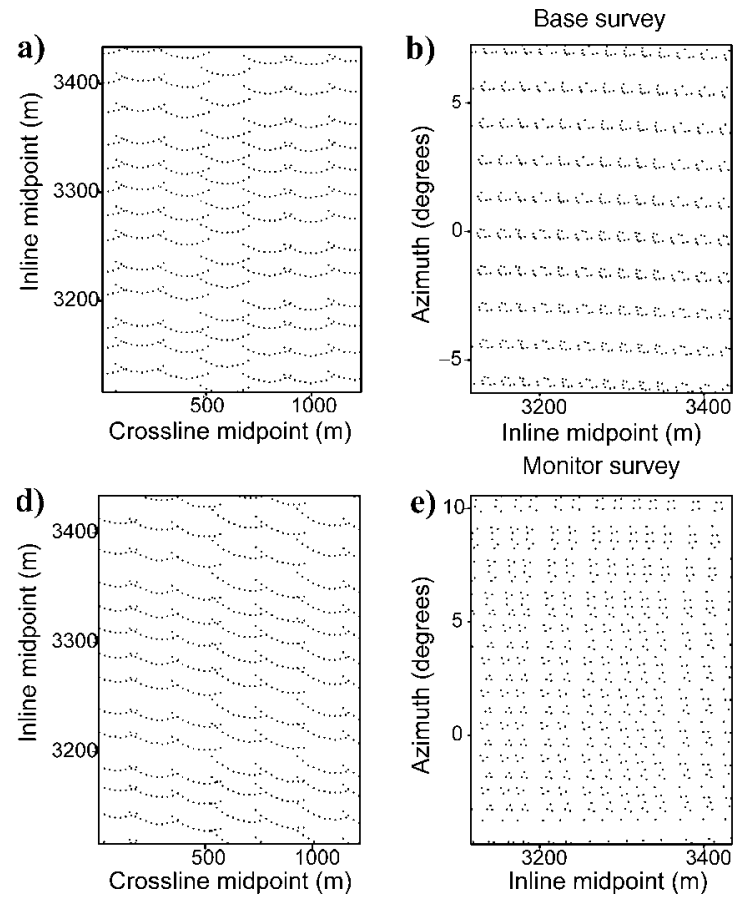
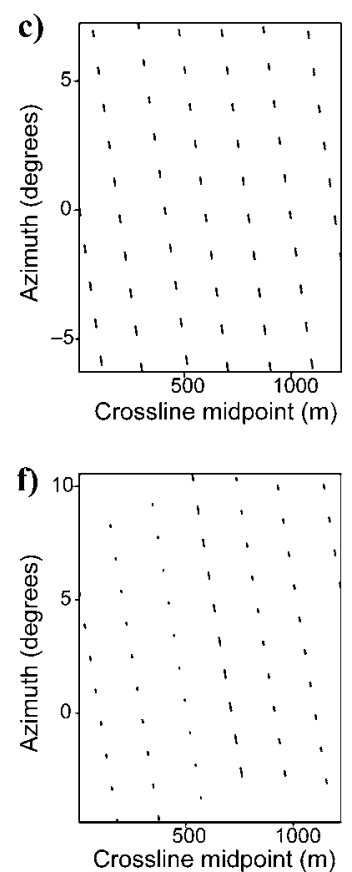

Figure 8. (a-c) Crossline midpoint, inline midpoint and azimuth sampling in a common-offset volume of $2000 \mathrm{~m}$ for the base data set; (d-f) idem for the monitor data set.
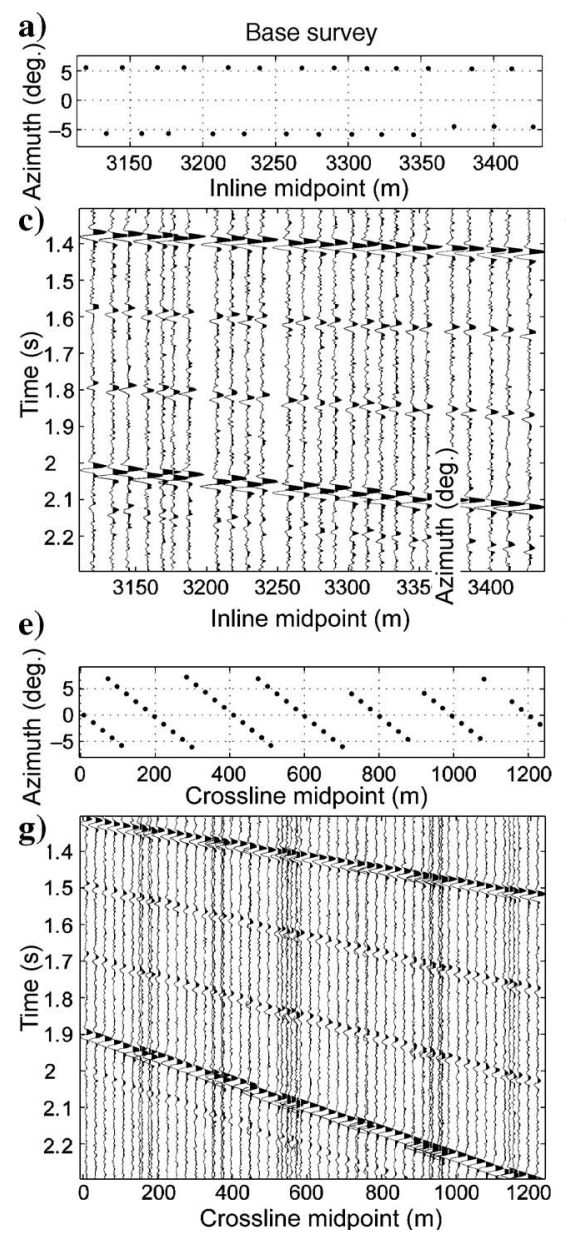

b)

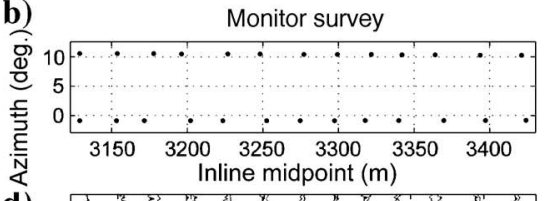

d)

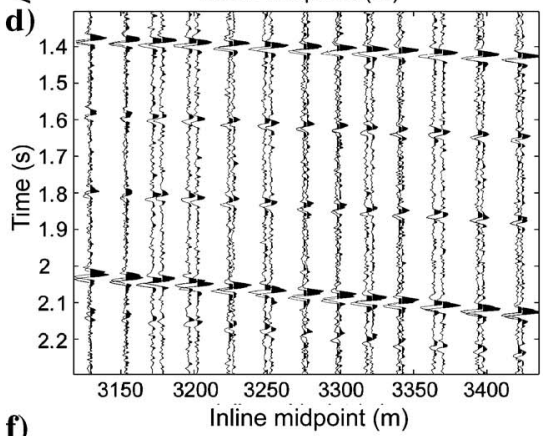

f)

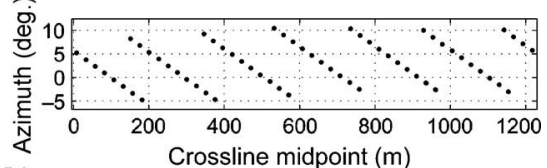

h)

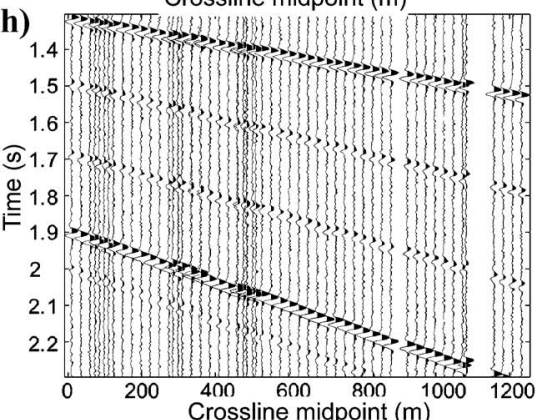

Figure 9. (a-d) Noisy common crossline and (e-h) common inline midpoint gathers from the synthetic base (left column) and monitor surveys (right column). Above each gather, the azimuth versus inline/crossline midpoint coordinates are plotted. 
muthal coordinates between adjacent swaths, shown in Figure 9e and $\mathrm{f}$. One can expect larger traveltime variations in the common crossline gathers than on the common inline gathers because the data have a common offset of $2000 \mathrm{~m}$ and the major component of that distance is in the inline direction. The crossline offset is restricted to the half-width of a swath $(225 \mathrm{~m})$.

\section{Reconstruction settings}

Based on the maximum expected dip of the reflection events in the data, we used a velocity cone with $c=2500 \mathrm{~m} / \mathrm{s}$ to limit the number of parameters. The maximum inline and crossline dips in the data are almost equal and are about $45^{\circ}$. This is also true for the maximum wavenumber $k_{\max }=n_{k} \Delta k$, where $\Delta k \approx 2 \pi /\left(x_{\max }-x_{\min }\right)$ and where $n_{k}$ is the number of $k$-values. Because the range in inline and crossline coordinates is different, $\Delta k$ is different for each direction; therefore, also the number of estimated $k$-values will be different. For the 2D Fourier reconstruction, we have chosen a maximum of 25 positive and 10 negative $k_{y}$ values, and eight positive and negative $k_{x}$ values.

The $3 \mathrm{D}$ reconstruction uses both midpoint coordinates and azimuth. The number of $k$-values in the inline and crossline midpoints is the same as in the 2D FRSI application. The limited range in azimuths does not allow for much resolution in azimuthal Fourier coefficients. Furthermore, the coarse crossline sampling introduces the risk of aliasing in the $\left(k_{m_{y}}, k_{\phi}\right)$ domain. In fact, choosing $k_{\phi_{\max }}>2 \pi /\left(\phi_{\max }-\phi_{\min }\right)$ already introduces aliasing. From inspection of a few 3D frequency slices obtained with the NDFT, we have set $n_{k_{\phi}}$ $=2$ and $\Delta k_{\phi}=1 / 3 \times 2 \pi /\left(\phi_{\max }-\phi_{\min }\right)$ to avoid aliasing. As the offset increases in marinestreamer acquisition, the azimuthal aperture shrinks. Because the azimuthal traveltime effect increases with offsets, it would make sense to increase the maximum azimuthal wavenumber $k_{\phi_{m}}$. Because the resolution in the azimuthal aperture decreases with offset, we may increase $k_{\phi_{\max }}$ and $\Delta k_{\phi}$ so that the number of coefficients $n_{k_{\phi}}$ remains constant. The effect of increasing overlap will be to reduce aliasing problems along the azimuthal coordinate.

\section{Reconstruction results}

Figure 10 shows results for the reconstruction of the common-offset volume at $2000 \mathrm{~m}$ to uniform grid with inline and crossline midpoint sampling of $6.25 \mathrm{~m}$ and $25 \mathrm{~m}$ for both the $2 \mathrm{D}$ and $3 \mathrm{D}$ FRSI algorithm. The 3D FRSI output is to zero azimuth on both base and monitor data sets. The results look virtually identical, which could be expected because the azimuthal variation in the data is small and will stand out only when difference sections are made. The 2D FRSI algorithm executed in $15 \mathrm{~s}$, while the 3D FRSI algorithm ran for 670 s on one CPU of an SGI Origin 200.

Figure 11 shows the normalized rms (NRMS; Kragh and Christie, 2002) differences per trace and difference plots zoomed in on the deepest reflection for the 2D and 3D FRSI reconstructions (on the same scale as Figure 10). The 2D FRSI algorithm accounts only for the average azimuthal variation. Therefore, the reconstructed data will be slightly different for the base and monitor data sets, which yield the differences shown in Figure 11. The reconstruction error in 2D FRSI is largest on the inline gather, with an average NRMS of $80 \%$. The azimuth varies more with crossline midpoint than with inline midpoint, which explains the difference in NRMS values along the gather in Figure 11. The 3D FRSI reconstructionto zero azimuth shows lower overall NRMS values and less energy on the difference plots. The values are comparable for the inline and crossline gathers, which have an average NRMS error of $30 \%$ and $26 \%$, respectively.

Fourier reconstruction clearly benefits from allowing for azimuthal variation in the data. However, the fact that the difference is small does not necessarily mean that the reconstruction to zero azimuth is correct. Therefore, we modeled zero-azimuth data and compared this to the 2D FRSI and 3D FRSI base and monitor reconstruc-
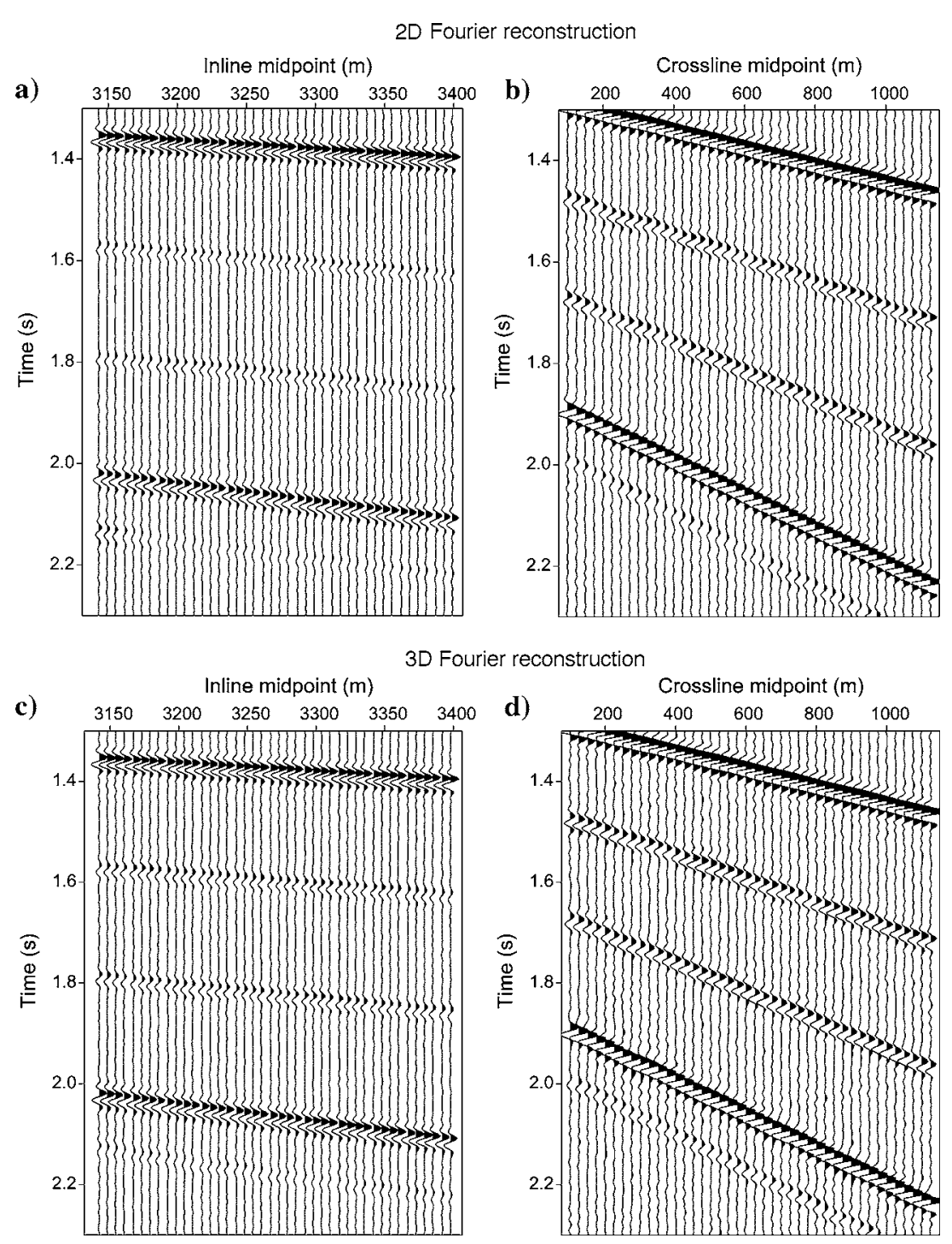

Figure 10. (a) Inline and (b) crossline section from 2D FRSI reconstruction; (c) inline and (d) crossline section from 3D FRSI reconstruction. 
tions. Figure 12 shows the NRMS plots and difference sections. Again, the difference is large for the 2D FRSI reconstruction because of averaging data for different azimuths. The 2D FRSI reconstruction shows a consistent and good quality reconstruction, which is good enough for structural imaging but not good enough for timelapse seismic processing. The 3D FRSI reconstruction shows a small difference compared to the modeled data, which indicates that the reconstruction to zero azimuth is performed correctly.

Figure 13 shows the result of the reconstruction of all azimuths between $+10^{\circ}$ and $-10^{\circ}$ at a particular midpoint. Because the azimuthal traveltime variation is small, there is only a small dip. The successful generation of data at different azimuths, within the aperture of the streamers and under appropriate conditions, suggests that the 3D FRSI algorithm can reconstruct data from source lines in be-
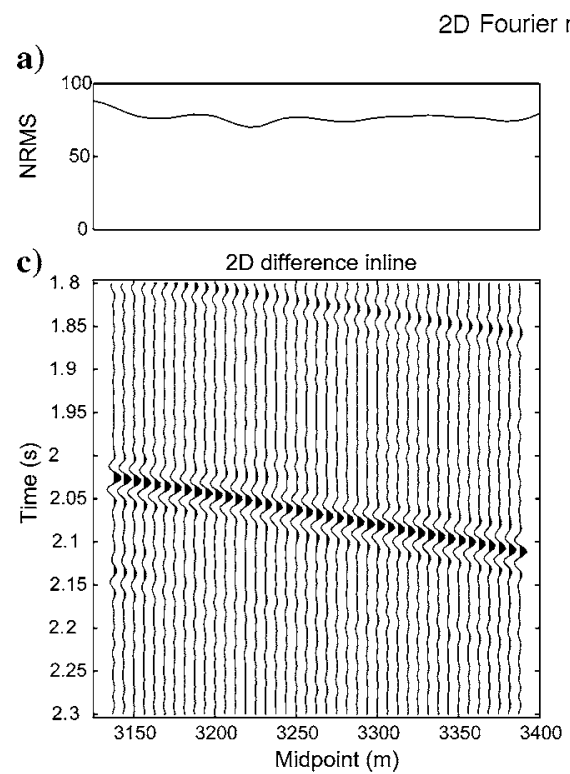

reconstruction

b)
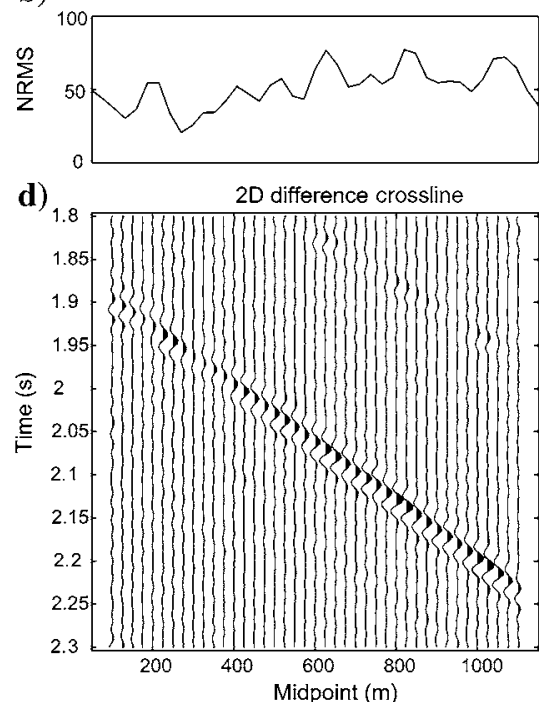

3D Fourier reconstruction
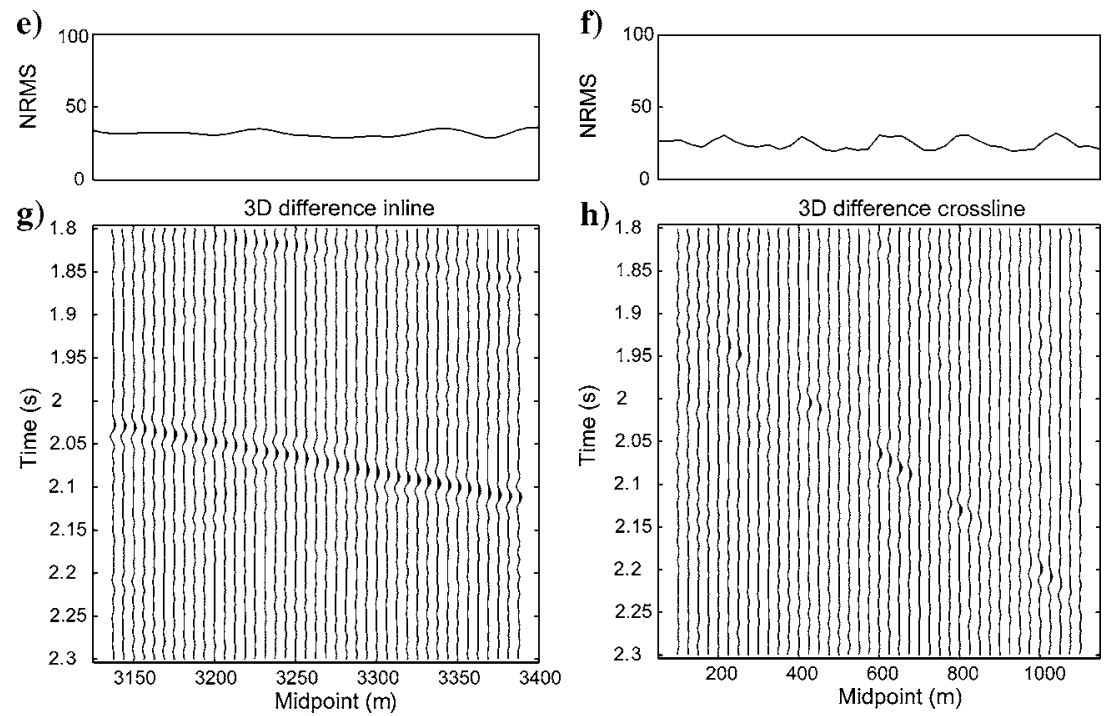

Figure 11. Difference sections between the reconstructed base and monitor surveys and normalized rms plot per trace for (a, c) inline gather with 2D FRSI; (b, d) crossline gather with 2D FRSI; (e, g) inline gather with 3D FRSI; (f, h) crossline gather with 3D FRSI. The gather is zoomed in on the deepest and steepest event. The amplitude in the difference section is on the same scale as the reconstructed data in Figure 10. tween swaths. A possible application for this is in 3D SRME, where missing source lines can be reconstructed to improve 3D multiple predictions, as Hokstad and Sollie (2004) do with a 2D linear Radon transform and sparse inversion. The advantage of 3D FRSI is improved handling of the azimuthal effects.

\section{DISCUSSION}

Our reconstruction procedure uses a combination of reconstruction in one and three spatial dimensions, and the main requirement is the sampling geometry must have more than single-azimuth multiplicity. The simple reason for this is that one cannot interpolate data in the azimuthal direction from a single measurement. This aspect should be taken into account when designing acquisition surveys, e.g., for time-lapse studies. If the azimuthal variation is not that strong and only varies significantly owing to discontinuities at the edge of the swath, then the sampling requirement is met already to a certain extent by having overlapping subsurface lines at the edges of a swath.

We have performed the reconstruction in the common inline midpoint domain and in the common-offset domain. The azimuthal traveltime variation is typically small and approximately linear within the aperture of streamer surveys. The azimuthal spatial bandwidth therefore will be generally small and can be limited to a few coefficients. The moveout and spatial sampling along the offset dimension are typically quite large, and one would certainly want to apply NMO to reduce the spatial bandwidth. In the common-offset domain, the bandwidth cannot be reduced by $\mathrm{NMO}$, but because the midpoint-sampling intervals are smaller, aliasing is less problematic. In both the common inline midpoint and commonoffset domains, the azimuthal traveltime effect can vary with the remaining two coordinates (crossline midpoint/offset and crossline/inline midpoint). However, in the common-inline-midpoint domain, the azimuthal traveltime variation generally increases with offset, requiring an offset-dependent azimuthal bandwidth. In the common-offset domain, the azimuthal traveltime variation as a function of inline midpoint is linked more directly to the subsurface, either to largescale stress regimes in case of azimuthal anisotropy or to smaller-scale structural effects.

Because interpolation along the azimuthal coordinate affects the crossline-source sampling, a potential application of the proposed method is to decrease the crossline source sampling for the benefit of 3D multiple prediction. Another application would be the regularization of time-lapse data to reduce the effects of sampling differences on time-lapse attributes. Also, there is a potential for application to nonuniformly sampled land data.

Before application to marine-streamer data, one would have to correct for spherical spreading and crossline timing issues attributable to tidal 
2D Fourier reconstruction

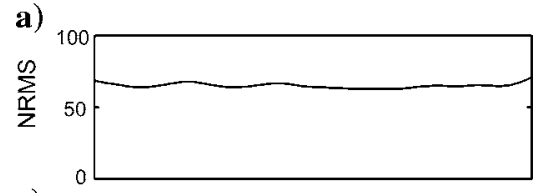

c)

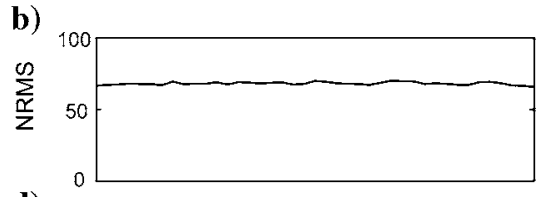

d)

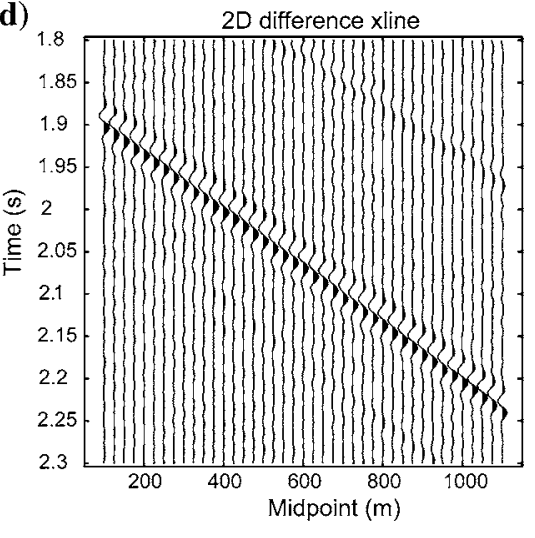

3D Fourier reconstruction
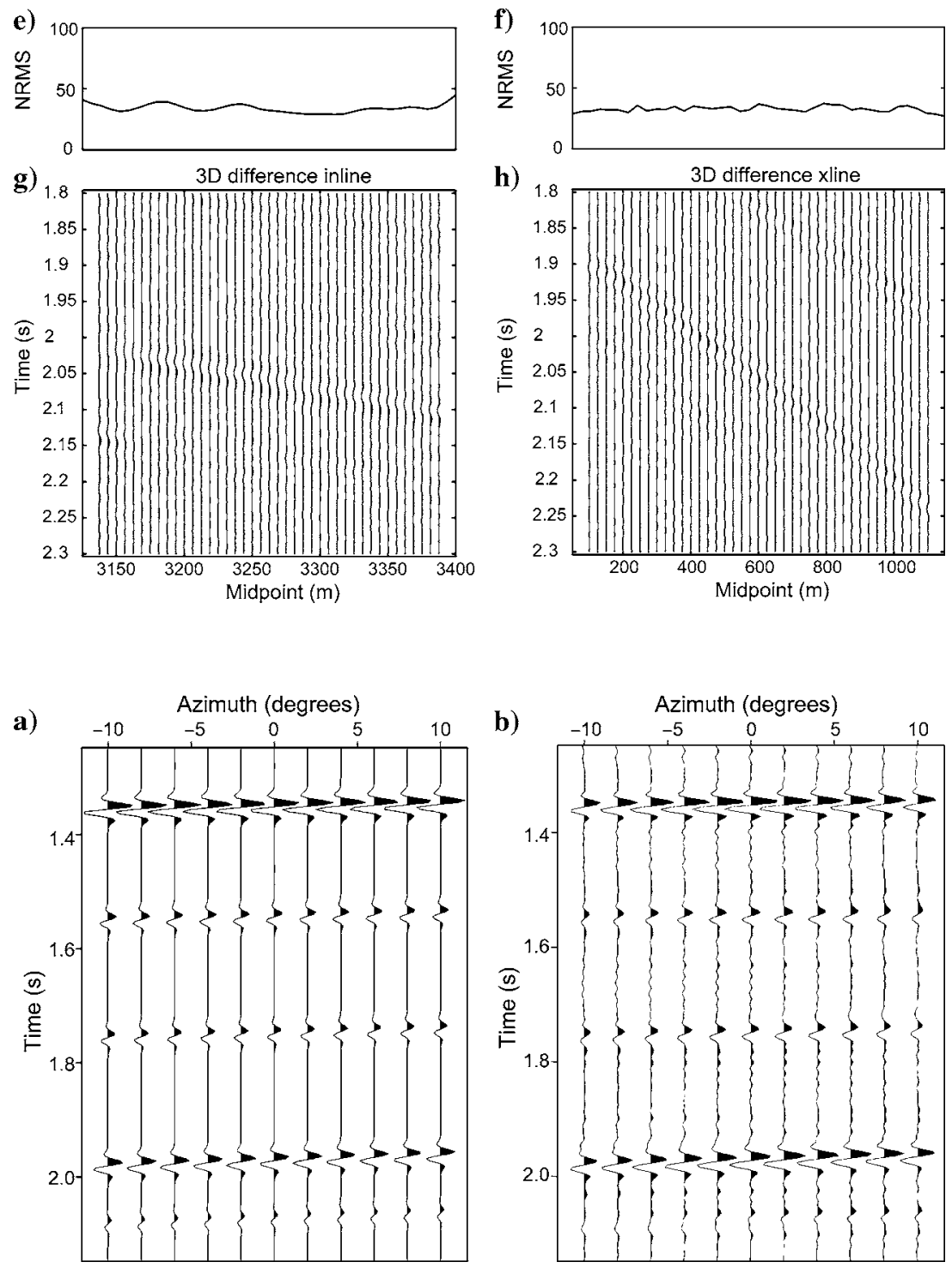

Figure 12. (a) Difference between modeled data at zero azimuth for the inline gather and 2D FRSI reconstruction; (b) idem for the crossline gather; (c) difference between modeled data at zero azimuth for the inline gather and 3D FRSI reconstruction. (d) Idem for the crossline gather. The amplitude in the difference section is on the same scale as the data in Figure 10.

Figure 13. (a) The 3D FRSI reconstruction to azimuths between $-10^{\circ}$ and $+10^{\circ}$ at a particular inline and crossline midpoint position; (b) modeled, noise-free data at the same position. 
and sail-line statics. Additionally, the method would benefit from application in spatio-temporal windows of, say, $40 \times 40 \mathrm{CMPs}$ and 500-1000 ms windows because this reduces the variability in the data. We have not verified the applicability of the method on field data; but given the interesting results obtained by Liu and Sacchi (2004), with a similar approach to real land data, we have positive expectations.

\section{CONCLUSION}

We have reconstructed subsets of synthetic marine-streamer data to test the feasibility of reconstruction in all four spatial coordinates. Our reconstruction procedure uses a combination of reconstructions in one and three spatial dimensions. Additional information required for the reconstruction of the sparsely sampled data is incorporated in the form of a sparseness constraint on the Fourier coefficients and is implemented via a nonquadratic model penalty function in the inversion. Good results have been obtained for nonuniformly sampled synthetic data generated using an anisotropic velocity model and a model containing dipping layers. The $3 \mathrm{D}$ inversion is computationally feasible because of the extension of the nonuniform FFT to three spatial dimensions and the use of a preconditioned conjugate gradient algorithm. Overall, the 3D FRSI algorithm is approximately an order of magnitude slower than 2D FRSI when applied to the same amount of data.

\section{ACKNOWLEDGMENTS}

The authors thank Mauricio Sacchi (University of Alberta) and Eric Verschuur (Delft University of Technology) for constructive discussions. We gratefully thank all the sponsors of DELPHI's Acquisition and Preprocessing Project for their financial support of this $\mathrm{Ph} . \mathrm{D}$. thesis research project.

\section{APPENDIX A \\ NONUNIFORM DISCRETE FOURIER TRANSFORM}

In Fourier reconstruction, the seismic data are reconstructed per temporal frequency. At each frequency $f$, the Fourier coefficients are estimated from the nonuniformly sampled data $p\left(x_{n}, f\right)$, where $x_{n}$ denotes the spatial coordinate of the $n$th trace. We will drop the dependence on $f$ for convenience. As a part of the inversion, we need an initial estimate of the Fourier coefficients, and this is provided by the nonuniform discrete Fourier transform. Also, it can be used as a starting model in the conjugate gradient scheme.

When the data $p\left(x_{n}\right)$ are nonuniformly sampled, we cannot use the fast Fourier transform to obtain the Fourier coefficients. Instead, we use the nonuniform fast Fourier transform (Duijndam and Schonewille, 1999a) to evaluate the nonuniform discrete Fourier transform (NDFT), which is defined as

$$
\begin{aligned}
& \tilde{p}\left(n_{k_{x}} \Delta k_{x}, n_{k_{y}} \Delta k_{y}, n_{k_{z}} \Delta k_{z}\right) \\
& \quad=\sum_{x_{i}} \sum_{y_{i}} \sum_{z_{i}} w_{i} p\left(x_{i}, y_{i}, z_{i}\right) e^{-j\left(n_{k_{x}} \Delta k_{x} x_{i}+n_{k_{y}} \Delta k_{y} y_{i}+n_{k_{z}} \Delta k_{z} z_{i}\right)},
\end{aligned}
$$

where $\left(x_{i}, y_{i}, z_{i}\right)$ denotes the location of the $i$ th trace and where $w_{i}$ are trace weights that compensate for differences in sampling density. The notation $\left(x_{i}, y_{i}, z_{i}\right)$ refers to any three coordinates and can be crossline midpoint, offset, and azimuth. For simplicity, the trace weights have been ignored in this paper. The integers $\left(n_{k_{x}}, n_{k_{y}}, n_{k_{z}}\right)$ denote the indices of the Fourier coefficients. The wavenumbersampling interval along the $k_{x}$ axis is defined as $\Delta k_{x}=2 \pi / X$, where the range in $x$ is $X=\left(x_{\max }-x_{\min }\right)$ with $\left(x_{\max }, x_{\min }\right)$ the maximum and minimum spatial coordinate along the $x$-axis. The wavenumber sampling intervals $k_{y}, k_{z}$ are defined in a similar manner.

The inverse transform from a uniformly sampled $k$-space to a nonuniformly sampled spatial grid is

$$
\begin{aligned}
p\left(x_{i}, y_{i}, z_{i}\right)= & \Delta S_{k} \sum_{n_{k_{x}}} \sum_{n_{k_{y}}} \sum_{n_{k_{z}}} \\
& \times \tilde{p}\left(n_{k_{x}} \Delta k_{x}, n_{k_{y}} \Delta k_{y}, n_{k_{z}} \Delta k_{z}\right) \\
& \times e^{-j\left(n_{k_{x}} \Delta k_{x} x_{i}+n_{k_{y}} \Delta k_{y} y_{i}+n_{k_{z}} \Delta k_{z} z_{i}\right)},
\end{aligned}
$$

where $\Delta S_{k}=\Delta k_{x} \Delta k_{y} \Delta k_{z} /\left(8 \pi^{3}\right)$. In vector notation, equation A-2 is written as $\mathbf{p}=\mathbf{A} \tilde{\mathbf{p}}$, where $\mathbf{p}$ contains the nonuniformly sampled data and the $\widetilde{\mathbf{p}}$ the Fourier coefficients. The inverse DFT is contained in the matrix $\mathbf{A}$.

\section{APPENDIX B}

\section{NONUNIFORM FAST FOURIER TRANSFORM}

In case of uniform sampling with missing samples, the NDFT yields the same coefficients as the DFT, with zeros inserted at the locations of the missing samples. This means the FFT can be used to compute the NDFT. Straightforward evaluation of the NDFT in equation A-1 is an $O(M N)$ operation, where $N$ is the number of input samples and $M$ the total number of Fourier coefficients. The NFFT is used to hasten the NDFT computations. This algorithm is presented by Duijndam and Schonewille (1999a); for details of the algorithm, we refer to that paper. In short, the algorithm works by convolving the nonuniformly sampled data with a window function of length $q$ along each dimension and specifying the output on a uniformly sampled grid that is oversampled by a factor $f$ with respect to the bandwidth of the input data. The now uniformly sampled data are FFT to the Fourier domain, followed by a correction for the convolution with the window function. We have extended this algorithm to three spatial dimensions (3D NFFT). The accuracy of the single-precision 3D NFFT and NDFT relative to double precision is defined as

$$
E_{2}(\beta)=\frac{\left\|\widetilde{p}_{\mathrm{NFFT}}-\widetilde{p}_{\mathrm{NDFT}}\right\|_{2}}{\left\|\widetilde{p}_{\mathrm{NDFT}}\right\|_{2}} .
$$

For an input signal consisting of $N=4000$ random numbers, the error $E_{2}$ with respect to the double-precision NDFT was computed for the single precision NDFT and a 3D NFFT with a truncated Gaussian and a Kaiser-Bessel window function. The results in Figure B-1 show that the error made by the single-precision NDFT has a constant value of about $1 e^{-6}$, whereas for both window function the error drops off rapidly as we increase the length $q$ of the filter. This experiment was run with the oversampling factor $f_{l}=2$ and $M_{l}=16$ Fourier coefficients for dimension $l$. 
The computation time is given approximately by

$$
t=c_{1} N \prod_{l=1}^{3} q_{l}+c_{2}\left(\prod_{l=1}^{3} f_{l} M_{l}\right) \sum_{l=1}^{3} \log f_{l} M_{l}+c_{3} \prod_{l=1}^{3} M_{l},
$$

where the first term on the right-hand side refers to the convolution in the spatial domain; the second term to the FFT; the third term to the window correction in the Fourier domain; and $c_{1}, c_{2}, c_{3}$ are processor-dependent constants (Duijndam and Schonewille, 1999a). This should be compared to the $O\left(N \prod_{l=1}^{3} M_{l}\right)$ operations for the direct evaluation of the NDFT. The computation time is evaluated for the 3D NFFT for different values of $M_{l}$ combined with $N=M_{l}^{3}$ and $N$ $=2 M_{l}^{3}$ for $q_{1}=8, f_{l}=2$ and $l=[1,2,3]$. The results shown in Table B-1 are for the NFFT with a Kaiser-Bessel window and a truncated Hanning window as convolution windows. We used $f_{l}=2$ and $q_{l}$ $=[3, \ldots, 10]$, where the subscript $l$ denotes the spatial dimension. At $f_{l}=2$ and $q_{l}=8$, the NFFT with the Kaiser-Bessel window is as accurate as the single-precision NDFT when applied to signals consisting of white noise. Of the filters with a closed-form expression, the

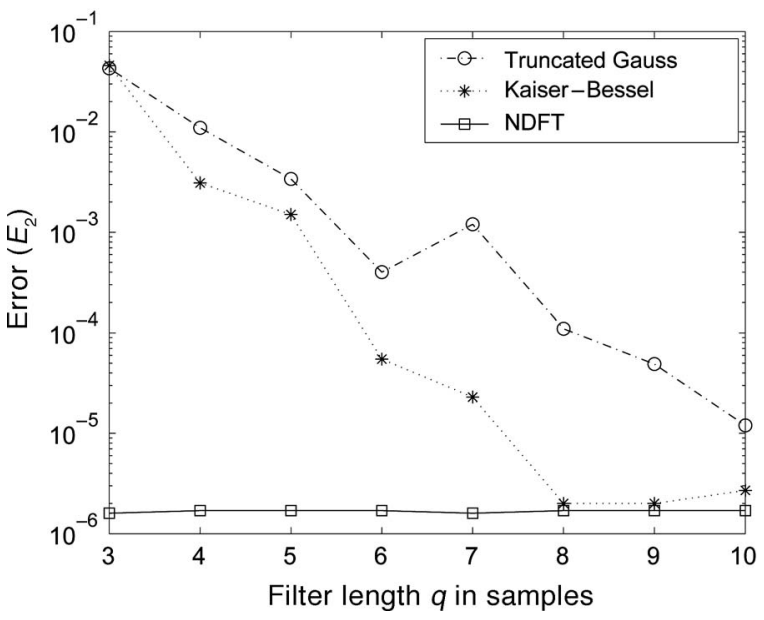

Figure B-1. The error $E_{2}$, as defined in equation B-1, between the double-precision NDFT and the single-precision 3D NFFT using a truncated Gaussian and a Kaiser-Bessel window function.

Table B-1. The computation times of the 3D NFFT for an eight-point Kaiser-Bessel window and an oversampling factor of two in each spatial dimension compared to the times for the 3D NDFT for various values of $M$ (number of Fourier coefficients in each dimension) and $N$ (number of samples).

\begin{tabular}{rrrrrr} 
& & \multicolumn{3}{c}{ Times for NFFT(s) } & \\
\cline { 3 - 5 }$M$ & $N$ & Init & Eval & Sum & $\begin{array}{c}\text { Times for } \\
\text { NDFT(s) }\end{array}$ \\
\hline 10 & 1000 & 0.14 & 0.06 & 0.20 & 0.2 \\
10 & 2000 & 0.16 & 0.12 & 0.28 & 0.4 \\
20 & 8000 & 0.78 & 0.52 & 1.30 & 8.3 \\
20 & 16,000 & 1.30 & 0.97 & 2.27 & 16.5 \\
30 & 27,000 & 2.56 & 1.79 & 4.35 & 94.0 \\
30 & 54,000 & 4.40 & 3.41 & 7.81 & 188.1 \\
\hline
\end{tabular}

Kaiser-Bessel window gives optimal results. The 1D discrete version is

$c(x)=\left\{\begin{array}{c}\frac{1}{I_{0}(\beta)} I_{0}\left(\beta \sqrt{1-\left(\frac{2 x}{q \Delta x}\right)^{2}}\right)-\frac{q \Delta x}{2} \leq x \leq \frac{q \Delta x}{2} \\ 0 \quad \text { otherwise }\end{array}\right.$

where $I_{0}$ is the zero-order modified Bessel function of the first kind. The Fourier transform of equation B-3 is

$$
\widetilde{c}(m)=\frac{\sin \sqrt{(\pi q \Delta x m)^{2}-\beta^{2}}}{\sqrt{(\pi q \Delta x m)^{2}-\beta^{2}}}=\frac{\sin \sqrt{(\pi q m /(f M))^{2}-\beta^{2}}}{\sqrt{(\pi q m /(f M))^{2}-\beta^{2}}},
$$

with $-M / 2 \leq m \leq M / 2-1$. The parameter $\beta$ affects the window shape. The optimal value for $\beta$ minimizes the relative error. We calculated this error for 100 realizations uniformly distributed random numbers and a range of $[q, f, \beta]$ and then averaged the results. The optimal value of $\beta$ for $q=10$ and $f=2$ can be seen in Figure B-2
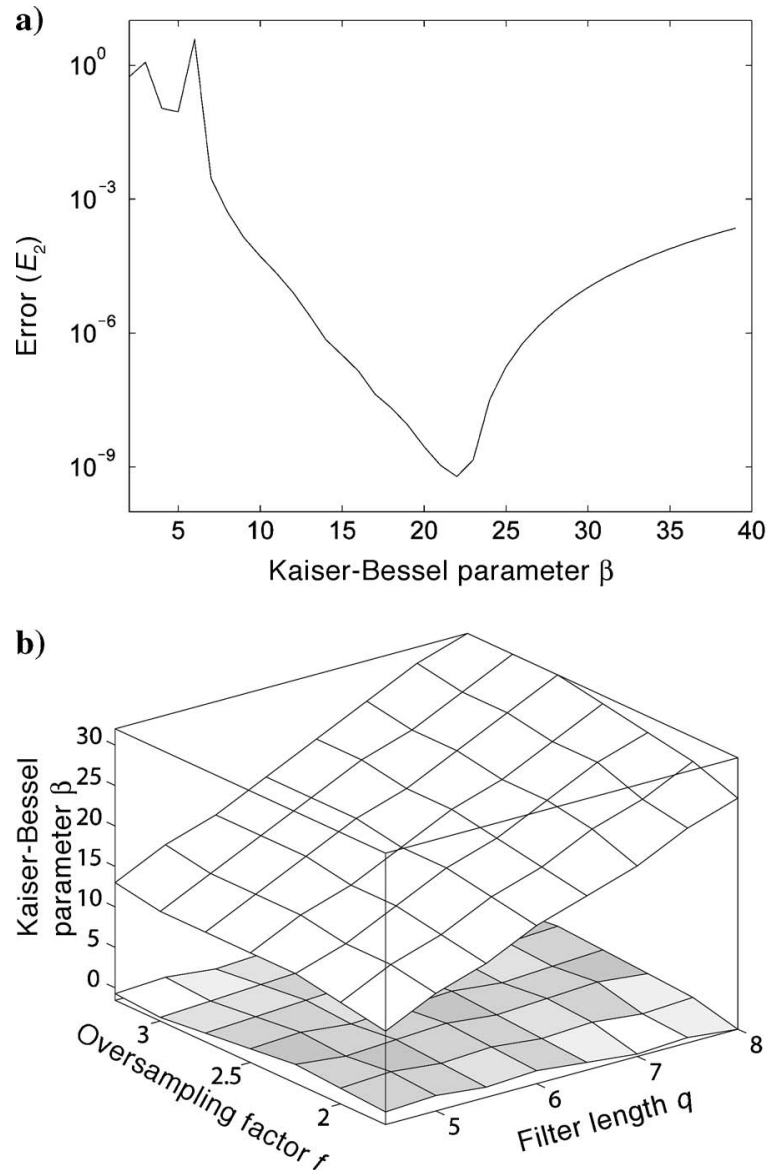

Figure B-2. (a) Reconstruction error $E_{2}$ as function of Kaiser-Bessel window parameter $\beta$ for $q=10$ and $f=2$. (b) The diagonal plane represents the optimal value of $\beta$ for the oversampling factor $f$ in the range $[2,3.5]$ and number of filter points $q$ in the range $[4,8]$. Equation B-5 represents a plane fit to these values. The horizontal plane shows the error between the plane fit and the optimal value of $\beta$, which is close to zero for all $q$ and $f$. 
a. An empirical relation for the optimal value of $\beta$ as a function of $q$ and $f$ was found to be

$$
\beta=2.68 q+1.27 f-6.14,
$$

as shown in Figure B-2b.

\section{REFERENCES}

Bardan, V., 1987, Trace interpolation in seismic data-processing: Geophysical Prospecting, 38, 343-358.

Baumstein, A., 2004, 3d inverse shot record dmo: A tool for shot-domain data regularization: 74th Annual International Meeting, SEG, Expanded Abstracts, 2001-2004.

Biondi, B., S. Fomel, and N. Chemingui, 1998, Azimuth moveout for 3D prestack imaging: Geophysics, 63, 574-588

Canning, A., and G. Gardner, 1998, Reducing 3D acquisition footprint for 3D DMO and 3D prestack migration: Geophysics, 63, 1177-1183.

Chemingui, N., and A. Baumstein, 2000, Handling azimuth variations in multistreamer marine surveys: 70th Annual International Meeting, SEG, Expanded Abstracts, $1-4$.

Duijndam, A. J. W., and M. A. Schonewille, 1999a, Non-uniform fast Fourier transform: Geophysics, 64, 539-551.

Duijndam, A. J. W., M., A. Schonewille, and C. O. H. Hindriks, 1999b, Reconstruction of band-limited signals, irregularly sampled along one spatial direction: Geophysics, 64, 524-538.

Eiken, O., G. U. Haugen, M. Schonewille, and A. Duijndam, 2003, A proven method for acquiring highly repeatable towed streamer seismic data: Geophysics, 68, 1303-1309.

Fomel, S., 2000, Three-dimensional seismic data regularization: Ph.D. thesis, Stanford University.

Hindriks, K., and A. Duijndam, 2000, Reconstruction of 3D seismic signals irregularly sampled along two spatial coordinates: Geophysics, 65, 253263.

Hokstad, K., and R. Sollie, 2004, 3D surface-related multiple elimination with source-line reconstruction: 74th Annual International Meeting, SEG, Expanded Abstracts, 1261-1264.

Hugonnet, P., and G. Canadas, 1997, Regridding of irregular data using 3D Radon decompositions: 67th Annual International Meeting, SEG, Expanded Abstracts, 1111-1114.

Kabir, M. M. N., and D. J. Verschuur, 1995, Restoration of missing offsets by parabolic Radon transform: Geophysical Prospecting, 43, 347-368.

Koek, E. A., and L. Ongkiehong, 1997, Regularization of 3D seismic data in two steps: 67th Annual International Meeting, SEG, Expanded Abstracts, $1171-1172$.

Kragh, E., and P. Christie, 2002, Seismic repeatability, normalized RMS and predictability: The Leading Edge, 21, 640-647.

Liu, B., and M. Sacchi, 2004, Minimum weighted norm interpolation of seismic records: Geophysics, 69, 1560-1568.

Liu, B., M. Sacchi, and H. Kuehl, 2003, 2D/3D seismic wavefield reconstruction for AVA imaging: 73rd Annual International Meeting, SEG, Expanded Abstracts, 235-238

Liu, B., M. D. Sacchi, and D. Trad, 2004b, Simultaneous interpolation of 4 spatial dimensions: 74th Annual International Meeting, SEG, Expanded Abstracts, 2009-2012.

Nemeth, T., W. Chengjun, and G. T. Schuster, 1999, Least-squares migration of incomplete reflection data: Geophysics, 64, 208-221.

Ronen, J., 1987, Wave-equation trace interpolation: Geophysics, 52, 973 984.

Sacchi, M. D., and T. J. Ulrych, 1995, Aperture compensated discrete Fourier transform and applications: 57th Annual International Meeting, EAEG, Session P071.

Schonewille, M., 2003, Regularization with azimuth time-shift correction: 73rd Annual International Meeting, SEG, Expanded Abstracts, 19171920.

Schonewille, M., R. Romijn, A. Duijndam, and L. Ongkiehong, 2003, A general reconstruction scheme for dominant azimuth 3D seismic data: Geophysics, 68, 2092-2105.

Spitz, S., 1991, Seismic trace interpolation in the F-X domain: Geophysics, 56, 785-794.

Trad, D., T. Ulrych, and M. Sacchi, 2003, Latest views of the sparse Radon transform: Geophysics, 68, 386-399.

Widmaier, M.. S. Hegna, F. Smit, and E. Tijdens, 2003, Azimuth preservation in marine 4D acquisition: 65th Annual International Meeting, EAGE, Expanded Abstracts, C04.

Xu, S., Y. Zhang, D. Pham, and G. Lambare, 2005, Antileakage Fourier transform for seismic data regularization: Geophysics, 70, V87-V95.

Zwartjes, P. M., 2005, Fourier reconstruction with sparse inversion: Ph.D. thesis, Delft University of Technology.

Zwartjes, P. M., and A. J. W. Duijndam, 2000, Optimizing reconstruction for sparse spatial sampling: 70th Annual International Meeting, SEG, Expanded Abstracts, 2162-2165.

Zwartjes, P. M., and M. Sacchi, 2004, Fourier reconstruction of non-uniformly sampled, aliased data: 74th Annual International Meeting, SEG, Expanded Abstracts, 1997-2000. 